\title{
Identification of new members of the MAPK gene family in plants shows diverse conserved domains and novel activation loop variants
}

\author{
Tapan Kumar Mohanta ${ }^{1 *}$, Pankaj Kumar Arora ${ }^{1}$, Nibedita Mohanta ${ }^{2}$, Pratap Parida ${ }^{3}$ and Hanhong Bae ${ }^{1 *}$
}

\begin{abstract}
Background: Mitogen Activated Protein Kinase (MAPK) signaling is of critical importance in plants and other eukaryotic organisms. The MAPK cascade plays an indispensible role in the growth and development of plants, as well as in biotic and abiotic stress responses. The MAPKs are constitute the most downstream module of the three tier MAPK cascade and are phosphorylated by upstream MAP kinase kinases (MAPKK), which are in turn are phosphorylated by MAP kinase kinase kinase (MAPKKK). The MAPKs play pivotal roles in regulation of many cytoplasmic and nuclear substrates, thus regulating several biological processes.

Results: A total of 589 MAPKs genes were identified from the genome wide analysis of 40 species. The sequence analysis has revealed the presence of several $\mathrm{N}$ - and C-terminal conserved domains. The MAPKs were previously believed to be characterized by the presence of TEY/TDY activation loop motifs. The present study showed that, in addition to presence of activation loop TEY/TDY motifs, MAPKs are also contain MEY, TEM, TQM, TRM, TVY, TSY, TEC and TQY activation loop motifs. Phylogenetic analysis of all predicted MAPKs were clustered into six different groups (group A, B, C, D, E and F), and all predicted MAPKs were assigned with specific names based on their orthology based evolutionary relationships with Arabidopsis or Oryza MAPKs.
\end{abstract}

Conclusion: We conducted global analysis of the MAPK gene family of plants from lower eukaryotes to higher eukaryotes and analyzed their genomic and evolutionary aspects. Our study showed the presence of several new activation loop motifs and diverse conserved domains in MAPKs. Advance study of newly identified activation loop motifs can provide further information regarding the downstream signaling cascade activated in response to a wide array of stress conditions, as well as plant growth and development.

Keywords: Mitogen activated protein kinase (MAPK), Activation loop, Conserved motifs, Orthologs, Paralogs, Phylogeny, Evolution

\section{Background}

During evolution, plants have developed complex arrays of defense mechanisms to mitigate the copious, often adverse and ever changing environmental conditions. Perception of variations in environmental as well as internal developmental cues, transduction and amplification of signals and activation of the response to stimuli is crucial for survival, optimal growth and development. Protein kinases are important signaling molecules that perceive various signals and transduce them for active

\footnotetext{
* Correspondence: nostoc.tapan@gmail.com; hanhongbae@ynu.ac.kr 'School of Biotechnology, Yeungnam University, Daehak Gyeongsan, Gyeonsangbook 712749, Republic of Korea

Full list of author information is available at the end of the article
}

responses. These compounds carryout diverse phosphorylation processes at the transcriptional, translational and post-translational level by catalyzing the addition of phosphate groups to serine and threonine/tyrosine residues in their target proteins in both prokaryotic and eukaryotic cells [1,2]. These modifications have led to changes in catalytic activity, affinity and interaction activity of target protein. However, the phosphorylation events in proteins are reversible due to protein phosphatase, enabling maintenance of the balance between kinase driven phosphorylation and phosphatase driven dephosphorylation events [3].

Plant genomes are rich in genes that encodes protein kinases and constitute the kinase super-family [4]. These 
super families are divided into different classes based on amino acid sequence similarity and functional characteristics. The mitogen activated protein kinase gene family, which is one such family, is known for evolutionary conservation across eukaryotic taxonomic groups and functioning within hierarchical cascades [1]. Phosphorylated proteins carry out a wide array of cellular responses, including changes in gene expression, innate immunity, developmental programmes and stress and hormonal responses [5-8].

Mitogen activated protein kinases consist of three kinasemodules composed of mitogen activated protein kinase kinase kinase (MAPKKKs), mitogen activated protein kinase kinase (MAPKKs) and mitogen activated protein kinase (MAPKs). In the general model, extracellular signals activate MAPKKKs, which phosphorylate downstream MAPKKs. The phosphorylated MAPKKs in turn phosphorylate MAPKs $[9,10]$. Protein phosphorylation events may occur throughout the protein kinase sequences, but usually occur on the activation loop [11]. The activation loop, which is present at the $\mathrm{C}$-terminal end, resides within sub-domain VII and VIII of sub-domain eleven [4]. The activation loop contains conserved serine, threonine and/or tyrosine amino acid residues that may be reversibly phosphorylated [6] via cis auto-phosphorylation or trans phosphorylation by upstream kinases [12].

The initial descriptions of components of the MAPK cascade have been provided for the popular model plant, Arabidopsis. Advancements in sequencing technologies and bioinformatics tools have greatly increased the pace of genome sequencing projects, resulting in successful sequencing of several plant genomes. Post genome sequencing projects have enabled relatively easy identification of particular gene families based on conserved signature motifs and sequence similarity. Available genome sequences from several plants genomes have provided us with an opportunity to identify MAPK family members across photosynthetic eukaryotes (plants and algae) that will shed more light on MAPK evolution and signaling in plants and lower photosynthetic eukaryotes. In recent years, identification of MAPK gene family members in plants has been limited to a few species including Arabidopsis thaliana [13], Oryza sativa [14], maize [15], Brassica napus [16], apple [17], and Brachypodium [18]. Further, a study by Janitza et al. [19] and a review article by Doczi et al. [20] have provided a comprehensive overview of the evolutionary history of MAPKs in green plants by using a limited number of plants species.

However, there is currently limited information regarding the nomenclature, conserved structures, genomics and biochemistry of MAPKs in plants. In this communication, we identify the MAPK gene families of 40 different plant species and provide a unique nomenclature to all MAPKs. This nomenclature system can be further applied to newly identified MAPKs of other species. Furthermore, the genomics, biochemistry and conserved consensuses of plant MAPKs describe several novel aspects of plant MAPKs.

\section{Results}

Identification and nomenclature of MAPKs

We identified the MAPK gene family from 40 different plant species starting from the unicellular lower eukaryote Chlamydomonas reinhardtii to the multi-cellular angiosperm Arabidopsis thaliana and attempted to cover the maximum number of species across the plant lineage. We found that MAPKs members of a genome varied from species to species across the whole plant lineage. The 40 species collectively gave rise to 589 MAPK sequences. The tetraploid Glycine max contained the most MAPK genes in its genome (31), whereas the lower eukaryotic plant Ostreocccus lucimarinus contained three (Table 1). In addition, Brassica compestris (30), Gossipium raimonddi (28), Malus domestica (28), Panicum virgatum (27), Linum usitatissimum (24) and Populus trichocarpa (21) contained higher number of MAPKs (Table 1, Additional file 1). All the identified 589 MAPKs were provided with specific names according to the orthologous sequence similarity with Arabidopsis thaliana or Oryza sativa.

\section{Genomics of MAPKs}

Among 589 MAPKs identified from 40 different plant species, Fragaria vesca FvMPK20 contains the largest MAPK gene, with 2574 nucleotides long open reading frame (ORF), while Panicum virgatum PvMPK1-4 has the smallest gene, with 544 nucleotides long ORF (Additional file 1). Transcript organization showed that MAPKs have different arrays of intron organization in their genes. The numbers of MAPKs containing different arrays of introns were as follows: intronless (7), single intron (41), two introns (39), three introns (18), four introns (15), five introns (161), six introns (32), seven introns (20), eight introns (39), nine introns (126), ten introns (72) and eleven introns (18)(Additional file 1). The terrestrial plant Selaginella moellendorffii SmMPK10 contained maximum of 14 introns in its gene. Some intronless MAPKs present in higher eukaryotic plants include PvMPK7-2, PaMPK2, PaMPK3, PaMPK7-1, and PaMPK20, while lower eukaryotic algae contain OlMPK7 and OlMPK9 (Additional file 1).

The molecular weights of MAPK proteins were vary from 22.381 (VvMPK1) to 98.915 (MdMPK20-2) kDa and the isoelectric points vary from 5.00 (MdMPK20-1) to 9.52 (CsubMPK15) (Additional file 2). The isoelectric point (pI) of group A and group B MAPKs were ranges from acidic to slightly acidic, while those of group $C$ and group D were reside within the basic $\mathrm{pI}$ ranges. The average amino acid composition of MAPK protein showed that, abundance of leucine (9.63) amino acid was maximum and tryptophan (0.70) amino acid was minimum 
Table 1 Table representing genome size of different plant species and number of MAPK genes present per genome (species)

\begin{tabular}{|c|c|c|c|c|c|c|c|}
\hline $\begin{array}{l}\text { Sl. } \\
\text { No }\end{array}$ & $\begin{array}{l}\text { Name of plant } \\
\text { species }\end{array}$ & $\begin{array}{l}\text { Abbreviation of } \\
\text { MAPKs }\end{array}$ & $\begin{array}{l}\text { Type of } \\
\text { organism }\end{array}$ & $\begin{array}{l}\text { Ploidy } \\
\text { level }\end{array}$ & $\begin{array}{l}\text { Genome size } \\
\text { (Mbs) }\end{array}$ & $\begin{array}{l}\text { Total No. of } \\
\text { loci }\end{array}$ & $\begin{array}{l}\text { Total No. of MAPK } \\
\text { genes }\end{array}$ \\
\hline 1 & Aquilegia coerulea & AcMPK & Dicot & Diploid & 302 & 24823 & 10 \\
\hline 2 & Arabidopsis thaliana & AtMPK & Dicot & Diploid & 135 & 27416 & 20 \\
\hline 3 & $\begin{array}{l}\text { Brachipodium } \\
\text { distachyon }\end{array}$ & BdMPK & Monocot & Diploid & 272 & 26552 & 16 \\
\hline 4 & Brassica rapa & BrMPK & Dicot & Diploid & 283.8 & 26374 & 30 \\
\hline 5 & Capsella rubella & CrMPK & Dicot & Diploid & 134.8 & 26521 & 18 \\
\hline 6 & Carica papaya & CpMPK & Dicot & Diploid & 135 & 27332 & 9 \\
\hline 7 & $\begin{array}{l}\text { Chlamydomonas } \\
\text { reinhardtii }\end{array}$ & CreinMPK & Algae & Haploid & $111+7.8$ & 12264 & 6 \\
\hline 8 & Citrus clememtina & CCMPK & Dicot & Diploid & 301.4 & 24533 & 12 \\
\hline 9 & Citrus sinensis & CsMPK & Dicot & Diploid & 319 & 25376 & 12 \\
\hline 10 & $\begin{array}{l}\text { Coccomyxa } \\
\text { subellipsoidea }\end{array}$ & CsubMPK & Algae & Haploid & 49 & 9629 & 4 \\
\hline 11 & Cucumis sativus & CsMPK & Dicot & Diploid & 203 & 21494 & 14 \\
\hline 12 & Eucalyptus grandis & EgMPK & Dicot & Diploid & 691 & 36376 & 13 \\
\hline 13 & Fragaria vesca & FVMPK & Dicot & Diploid & 240 & 32831 & 11 \\
\hline 14 & Glycine max & GmMPK & Dicot & Tetraploid & 975 & 54175 & 31 \\
\hline 15 & Gossipium raimondi & GrMPK & Dicot & Diploid & 761.4 & 37505 & 28 \\
\hline 16 & Linum usitatissimum & LuMPK & Dicot & Diploid & 318.3 & 26374 & 24 \\
\hline 17 & Malus domestica & MdMPK & Dicot & Diploid & 881.3 & 26.374 & 28 \\
\hline 18 & Manihot esculenta & MeMPK & Dicot & Diploid & $533(760)$ & 30666 & 17 \\
\hline 19 & Medicago truncatula & MtMPK & Dicot & Diploid & $241+16.6$ & 44135 & 17 \\
\hline 20 & Micromonas pusila & MpMPK & Algae & Haploid & 22 & 10660 & 4 \\
\hline 21 & Mimulus guttatus & MgMPK & Dicot & Diploid & 321.7 & 26718 & 6 \\
\hline 22 & Oryza sativa & OsMPK & Monocot & Diploid & 372 & 39049 & 17 \\
\hline 23 & $\begin{array}{l}\text { Ostreococcus } \\
\text { lucimarinus }\end{array}$ & OIMPK & Algae & Haploid & 13.2 & 7796 & 3 \\
\hline 24 & Panicum virgatum & PVMPK & Monocot & Tetraploid & 1358 & 65878 & 27 \\
\hline 25 & Phaseolus vulgaris & PVMPK & Dicot & Diploid & 521.1 & 27197 & 14 \\
\hline 26 & Physcomitrella patens & PpMPK & Bryophyte & Haploid & 480 & 32272 & 8 \\
\hline 27 & Picea abies & PaMPK & Gymnosperm & Diploid & 1960 & 28354 & 14 \\
\hline 28 & Populus trichocarpa & PtMPK & Dicot & Diploid & 422.9 & 41335 & 21 \\
\hline 29 & Prunus persica & PperMPK & Dicot & Diploid & $227.3+224.6$ & 27864 & 12 \\
\hline 30 & Ricinus communis & RCMPK & Dicot & Diploid & 400 & 31221 & 12 \\
\hline 31 & $\begin{array}{l}\text { Selaginella } \\
\text { moellendorffii }\end{array}$ & SmMPK & Pteridophyte & Haploid & 212.5 & 22273 & 6 \\
\hline 32 & Setaria italica & SiMPK & Monocot & Diploid & 405.7 & 35471 & 16 \\
\hline 33 & Solanum lycopersicum & SIMPK & Dicot & Diploid & 900 & 34727 & 17 \\
\hline 34 & Solanum tuberosum & StMPK & Dicot & Diploid & 800 & 35119 & 12 \\
\hline 35 & Sorghum bicolor & SbMPK & Monocot & Diploid & 697.5 & 34496 & 16 \\
\hline 36 & Thellungiella halophila & ThMPK & Dicot & Diploid & 238.5 & 26351 & 16 \\
\hline 37 & Theobroma cacao & TCMPK & Dicot & Diploid & 330.8 & 29452 & 12 \\
\hline
\end{tabular}


Table 1 Table representing genome size of different plant species and number of MAPK genes present per genome (species) (Continued)

\begin{tabular}{|c|c|c|c|c|c|c|c|}
\hline 38 & Vitis venifera & VVMPK & Dicot & Diploid & 487 & 26346 & 12 \\
\hline 39 & Volvox carteri & VCMPK & Algae & Haploid & 125.4 & 14971 & 5 \\
\hline 40 & Zea mays & ZmMPK & Monocot & Diploid & 2500 & ?????? & 19 \\
\hline
\end{tabular}

From this table it is evident that, the number of genes in a specified gene family don't directly proportional to its genome size.

(Additional file 3). The average abundance of the most important amino acids threonine, glutamic acid, and tyrosine (T-E-Y) were 4.65, 6.73 and 3.91, respectively, whereas the average abundance of aspartic acid was 6.01. The abundance of the hydrophobic amino acids alanine (6.91), isoluecine (6.24), leucine (9.63), phenylalanine (4.45), proline (6.25) and valine (5.70) in MAPKs were relatively higher than that of other amino acids (Additional file 3).

\section{Conserved motifs and domains}

$\mathrm{N}$-terminal conserved sequences

The MAPKs are characterized by the presence of a conserved T-E-Y/T-D-Y motif in the activation loop region. Despite having the activation loop T-E-Y/T-D$\mathrm{Y}$ motif in MAPKs, in this study, we found that several MAPKs shared conserved N-terminal T-E-Y, TD-Y, S-D-Y and S-E-Y motifs (Figure 1A, 1B, Table 2, Additional file 4). These N-terminal conserved motifs are only shared by group D MAPKs. In total, 182 genes shared the N-terminal conserved motifs. Among them, 11 genes shared the S-D-Y motif, 27 shared the S-E-Y motif, six shared the T-D-Y motif and the remaining138 genes shared the T-E-Y motif (Additional file 4). Chlamydomonas and Volvox share a common A-V-H motif instead of the S-E-Y/ S-D-Y/T-E-Y and T-D-Y motif (Additional file 4). Several other group specific conserved motifs are also present in the N-terminal region of MAPKs. They includes A-K-Y, NK-Y (group A), S-K-Y, R-K-Y (group B), T-K-Y (group C) and S-Q-Y, N-R-Y, S-R-Y (group D) (Figure 2, Table 2). These motifs are present immediately after the N-terminal T-E-Y, T-D-Y, S-D-Y and S-E-Y motifs. The MAPK sequences sharing different numbers of motifs are A-K-Y (70), N-K-Y (13), S-K-Y (74), R-K-Y (42), T-K-Y (81), S-Q-Y (36), N-R-Y (98), and S-R-Y (91) (Additional file 4). In addition to the presence of conserved motifs, the N-terminal region of MAPKs also contained conserved amino acid consensus sequences including I-G-x-G-x-Y-G-x-V, I-K-K-I$x_{3}-F, D-A-x-R-x-L-R-E, F-x-D-I-Y-x_{3}-E-L-M, D-L-x_{2}-V-I, D-$ $x-L-x_{2}-E-H, Q-x-L-R-x-L-K-Y-x-H, H-R-D-L-K-P-x-N$, and L-X-L-x-N-C-x-L-K-I-x-D-F-G-L-A-R (Figure 1A, Table 3).

\section{Conserved sequences in activation loop region}

As discussed earlier, MAPKs contain the classic T-E-Y or T-D-Y motif in the activation loop region, and we found that majority of MAPK members contain classic
T-E-Y/ T-D-Y motif in the activation loop region. Sequence alignment revealed that $M$. domestica MdMPK9 and $P$. trichocarpa PtMPK17-1contain an additional sequence of TAYKQYFLWTKLLTFMKDY and TVCVFLK PGFTFQCLIDY between the conserved T-D-Y motif (Additional file 4). In this study, we found eight novel activation loop motifs of MAPKs and reported here for first time. The newly identified activation loop motifs are, T-Q-Y (group A), M-E-Y, T-E-C, T-V-Y, (group B), T-E-M (group D), T-S-Y, T-Q-M, and T-R-M (group E) (Figures 3 and 4 , Table 2). In total, eight MAPK genes share the M-E-Y motif in the classic T-E-Y and T-D-Y region (Additional file 4). The MAPK genes sharing the M-E-Y motif are $S$. lycopersicum SIMPK4-1, $S$. tubersum StMPK4-1, B. distachyon BdMPK4-2, P. vulgaris FvMPK4-2, S. italica SiMPK4-2, Z. mays ZmMPK42, S. bicolor SbMPK4-2 and O. sativa OsMPK16-2. These M-E-Y motifs fall under group B MAPKs. The new motif T-Q-Y (group A) is shared by BrMPK10-2, T-E-C (group B) by GrMPK4-6, T-V-Y (group B) by GmMPK4-1, T-E-M (group E) by PaMPK5, PaMPK14 and PaMPK7-2, while T-S-Y is shared by OlMPK7 (group E), T-Q-M (group E) by PaMPK10 and T-R-M (group E) by CsubMPK3 (Figure 3, Table 2).

\section{C-terminal conserved sequences}

The classical T-E-Y/T-D-Y motif at the activation loop region is followed closely by the presence of different C-terminal S-D-Y/S-E-Y/T-D-Y/D-N-Y/S-Q-Y/S-R-Y/SK-Y/S-N-Y motifs (Figure 3, Table 2). These S-D-Y/S-E$\mathrm{Y}$ and T-D-Y motifs are shared by group $\mathrm{A}$ and group $\mathrm{B}$ MAPKs. Additionally, the D-N-Y, S-Q-Y, and T-K-Y motifs are shared by group C MAPKs and S-K-Y, T-K-Y, S-R-Y, and S-N-Y motifs are shared by group D MAPKs (Table 2). The MAPK sequences sharing different numbers of C-terminal motifs include S-D-Y (57), S-E-Y (123), T-D-Y (8), D-N-Y (78), S-Q-Y (7), S-K-Y (217), S-R-Y (7), and S-N-Y (11). There are also conserved T-RW-Y-R-A-P-E-L,I-D-X-W-S-V-G-C and Q-X-L-L-X-F-D-P consensus sequences present in the immediately post activation loop region of MAPKs (Figure 1A, Table 3).

\section{Common docking domains}

During mitogen activated protein kinase (MAPK) signaling, the ability of MAP2Ks to recognize their cognate 


\section{A}

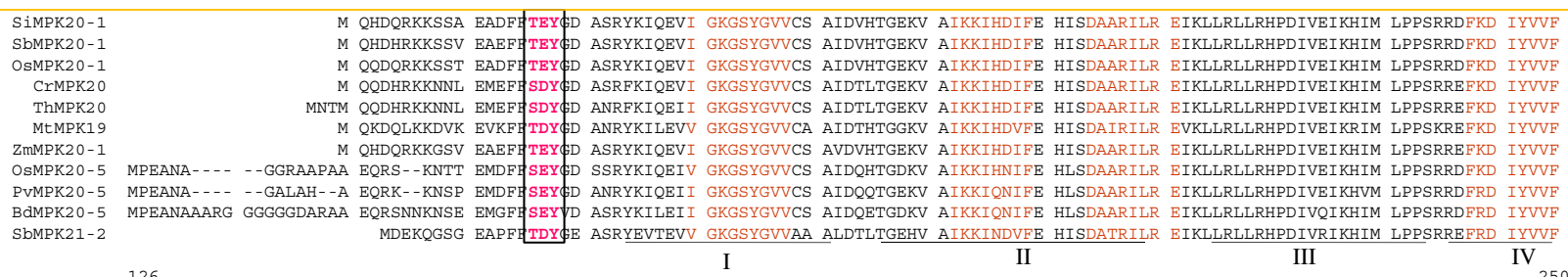

SIMPK20-1 ELMESDLHQV IKANDDLTKE HYQFFLYQLL RALKYIHTAN VYHRDLKPKN ILANSNCKLK ICDFGLARVA FSDTPTTIFW TDYVATRWYR APELCGSAFSKY PPAIDIWS IGCIFAEVLT GKPLF SbMPK20-1 ELMESDLHOV IKANDDLTKE HYOFFLYOLI RALKYIHTAN VYHRDLKPRN ILANSNCKLK ICDFGLARVA FNDTPTTIFW TDYYATRWYR APELCGSAFSKY PPAIDIWS IGCIFAEVLT GKPLF OSMPK20-1 ELMESDLHOV TKANDDLTKE HYQFFLYOLI RALKYTHTAN VYHRDLKPKN ILANSNCKL T ICDFGLARVA FNDTPTTIFW TDYVATRWYR APELCGSAFSKY TPAIDIWS IGCIFAEVLT GKPLF

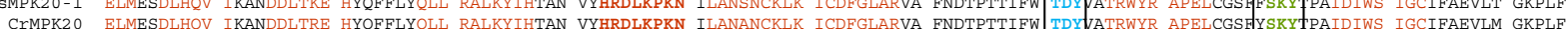
ThMPK20 ELMESDLHOV IKANDDLTRE HYOFFLYOLL RALKYIHTAN VYHRDLKPKN ILANANCKLK ICDFGLARVA FNDTPTTIFW TDYVATRWYR APELCGSAYSKY PPAIDIWS IGCIFAEVLM GKPLF MLMPK19 ELMESDLHOV IKANDDLTRE HOOFFLYOML RALKFMHTAN VYHRDLKPKN ILANANCKLK VCDFGLARVA FNDTPTTTFW TDYVATRWYR APELCGSAFAKY FPAIDIWS IGCIFAEVLT GKPLF ZMMPK20-1 ELMESDLHOV IKANDDLTKE HYOFFLYOLI RALKYTHTAN VYHRDLKPKN ILANSNCKLK ICDFGLARVA FNDTPTTIFW TDYVATRWYR APELCGSAFSKY PAAIDIWS IGCIFAEVVLT GKPLF OSMPK20-5 ELMDTDLHOV IKANDDITKE HHOFFLYOML RALKYIHTAN VYHRDLKPKN ILANANCKLK ICDFGLARVA FNDTPTTVFW TDYVATRWYR APELCGSAFSKY PVMPK20-5 ELMDTDLHOV IKANDDLTKE HHOFFLYOML RALKYIHTAN VYHRDLKPKN ILANANCKLK ICDFGLARVA FNDSPTTVFW TDYVATRWYR APELCGSAFTKY\$PAIDMWS IGCIFAEILT GKPLF BAMPK20-5 ELMDTDLHOV IKANDDLTKE HYQFFLYQML RALKYIHTAN VYHRDLKPKN ILANANCKLK ICDFGLARVA FNDTPTTVFW TDYVATRWYR APELCGSAFTKY PAIDTWS IGCIFAEILT GKPLF

SbMPK21-2 ELMESDLHOV IKANDDLTPE HHQFFLYQLL RGMKYIHAAN VFHRDLKPKN ILANGDCKLK ICDFGLARVS FNDTPSAIFW TDYYATRWYR APELCGSAFSKY PPAIDIWS IGCIFAEMLT GKPLF 251

$$
\mathrm{V}
$$

VII

\section{VIII}

375

SIMPK20 - 1 PGKNVVHOLD LMTDLLGTPS MDTISRVRNE KARRYLSSMR KKEPIPFSOK FPNADPLALD LLORLLAFDP KDRPTAEOAL AHPYFKSLAK VEREPSCQ-P ITKMEFEFER RRVTKEDIRE LIFRE SBMPK20-1 PGKNVVHOLD LMTDLLGTPS MDTISRVRNE KARRYLSSMR KKETISFSOK FPNADPLALD LLORLIAFDP KDRPTAEOAL AHPYFKGLAK VEREPSCO-P ITKIEFEFER RRVTKEDIRE IIFRE OSMPK20-1 PGKNVVHOLD LMTDLLGTPS MDTISRVRND KARRYLSSMR KKEPILFSOK FPSADPLALD LIOKLIAFDP KDRPTAEEAL AHPYFKGLLAK VEREPSCO-P ITKMEFEFER RRVTKEDIRE LIFR CrMPK20 PGKNVVHOLD LMTDLIGTPS LDTISRVRNE KARRYLTSMR KKPPIPFAOK FPNADPLSLK LLERT ThMPK20 PGKNVVNOLD LMTDLLGTPS LDTISRVRNE KARRYLTSMR KKPPIPFAOK FPNADPLSLK LLERLIAFDP KDRPTAEEAL ADPYFKGLAK VEREPSCQ-P ITKMEFEFER RKVTKEDIRE IISRE MLMPK19 PGKSIVHOLD LITDLLGTPP PEIISGVRNE KARKYLMEMR KKLPVPFFERK FPNADPLALR LLORLIAFDP KDRPTAQEAL ADPFFKGLAK IEREPSSQ-S ISRMEFEFER RRVSKDDIKE LIYRE ZMMPK20-1 PGKNVVHOLD LMTDLIGTPS MDTISRVRNE KARRYLSSMR KKETISFSOK FPHADPIALD LIORI A OSMPK20-5 PGKNVVHOLD LMTDLLGTPS MDAISRIRND KARRYLSSMR RKOPVPFSEK FPNVDPLALK LLORLIAFDP KDRPTAFEAL ADPYFKGLAK VEREPSCO-P ISKMEFEFER RKVTKDDIKE LIFRE PVMPK20-5 PGKNVVHOLD LMTDLIGTPS ADTISOIRNE KARRYLSSMR KKOPIPFSLK FPNADPSALK LLORLIAFDP KDRPTAEEAL ADPYFKGIAK VEREPSCQ-P ISKMEFEFER RKFTKEDVKE LIFO BAMPK20-5 PGKNVVHOLD LMTDLLGTPS TETISRIRND KARKYLSSMR RKOPIPFSEK FPNADPSALK LLERLLAFDP KDRPTAEEAL AHPYFKRLAR VEREPSCOOP ISKTEFEFER RKFTKEDVKE LIFRE SbMPK21-2 PGKNVVHQLD LMTDLLGTPS SESLSRIRNE KARRYLGNMR KKHPVPFTQK FPGIDPMALH LLERLIAFDP KDRPTAAEAL TDPYFTGLAN SEREPITQ-P ISKFEFEFER RKLARDDVRE LIYRE IX $\mathrm{X}$ XI

SIMPK20-1 ILEYHPQLLK DYINGTERTT FLYPSAVDQF RKQFAHLEEN SG-NGPVIPM ERKHTSLPRS TIVHSAPIPV KEQPRIGPSR EKPSSDESYK NSRETEKYSG NVSRT- -APQ R-VPTARPGR VVGPY SbMPK20-1 ILEYHPQLLK DYINGTERTT FLYPSAVDQF RKQFAHLEEN SG-NGPVIPM ERKHTSLPRS TIVHSAPIPV KEQPRIGPSR ERPSSDESYR NPRETEKYSG NLPRTSQAPQ R-VPTARPGR VVGPV OSMPK20-1 ILEYHPQLLK DYINGTERTT FLYPSAVDQF RKQFAHLEEN GG-NGPVIPM DRKHTSLPRS TIVHSTPIPA KEQPRIGPSR DKPS-DEPYS NPREFDRFSG NAPRTSQAPQ R-VPTARPGR VVGPV CrMPK20 ILEYHPQLLK DYMNGADKTS FLYPSAVDQF RRQFAHLEEN SGKSGPVAPL ERKHASLPRS TVIHSTAVAR GGQPKL- - - - - -MNNTNTM NPETTQNIPL NH-ATLQAPQ RNLSAAKPST FMGPV ThMPK20 ILEYHPQLLK DHMNGADKAS FLYPSAVDQF RRQFAHLEEN SGKTGPVAPL ERKHASLPRS TVIHSTAVAR GGPP-..... - .....--NFS SLETSQNVPL NHHASLQAPQ RNFSAAKPST FMGPV MtMPK19 ILEYHPQLLK DYINGTEGTN FMYPSAIDQF RKQFAYLEEN NGKCGPVIPP ERKHVSLPRS TVLSST-IPP STQSSFAPYA NRQIAQEAFS IPRAAESNSL SQSKGLRPPP R-APAAKSGR VTGPV ZmMPK20-1 ILEYHPQLLK DYINGTERTT FLYPSAVDQF RKQFAHLEEN SG-NGPVIPM ERKHTSLPRS TIVHSSPIPV KEQPRIGPCR ERPSSDESYR NPRETEQYSG NLPRTSQAPQ RVPTGTFGSC HVNMW OSMPK20-5 ILEYHPQLLK DYMNGSENTS FLYPSAVDNF RRQFAILEEN GGKSGAL--- DRKHVSLPRA TTVHSTSIPP NEGL--..- - -

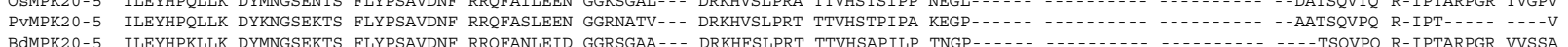
SbMPK21-2 TLEYHPQMLQ EFLGGGDKAN FVYPSGVDRF KRQFAHLEES AAKGEKTSPQ LRQHASLPRD RVVTNSSCIL SQGKSK

\section{B}
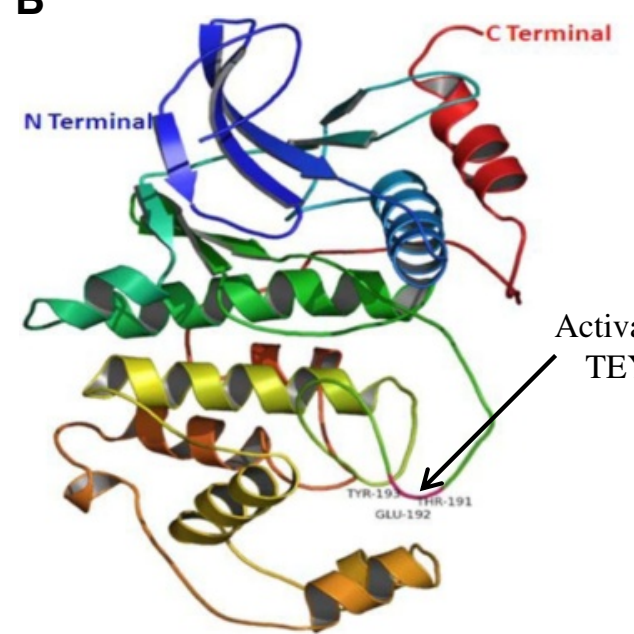

loop

Activation loop TDY Motif

TEY Motif

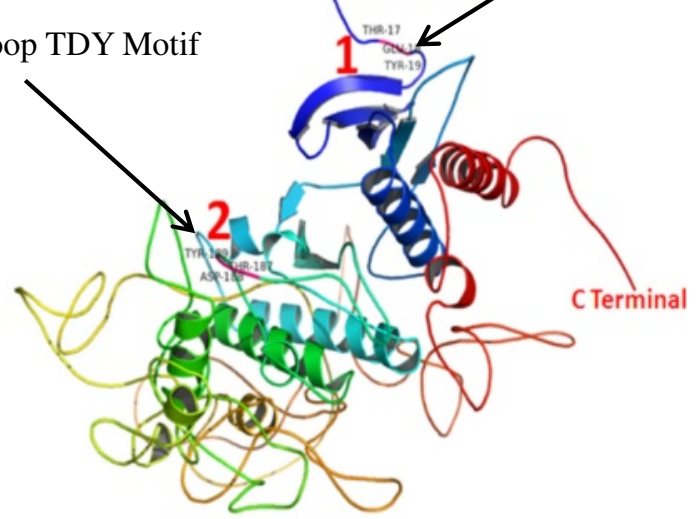

AtMPK1

SiMPK20-1

Figure 1 (See legend on next page.) 
(See figure on previous page.)

Figure 1 Multiple sequence alignment of plant MAPKs. A. The figure shows presence of N-terminal TEY, TDY, SEY and SDY motifs (in red). The SEY and SDY motifs are aligned with TEY and TDY motifs and very specific to group D MAP kinase genes. Activation loop TDY motifs are marked in blue and indicated inside the box. Conserved signature consensus sequences present within MAP kinases domain of MAPK proteins are marked in maroon. C-terminal conserved motifs are marked in green and presented inside box. All eleven sub-domains are also indicated in the figure. B. Molecular structure of AtMPK1 (group C) and SiMPK20-1 (group D). In AtMPK1, the arrow mark shows presence of TEY motif in the activation loop region. Similarly, in the group D MAP kinase (SiMPK20-1), the conserved N-terminal TEY motif and activation loop TDY motifs are indicated by different arrows.

MAPKs are facilitated by presence of short docking motif (D-site) that binds to its target complementary region on the MAPK. Similarly MAPKs are also contain short docking site that recognizes many downstream target proteins by utilizing the same strategy. From the studied MAPKs, we did not find presence of any unique and specific conserved docking domains for all groups of the MAPKs. Instead, conservation of the docking domains consensus is somewhat group specific (Table 4). The conserved docking domains of different MAPKs are K-M-L-TF-D-P-K/R-Q/K-R-I-T-V-E-D/E-A-L (group A), K-M-L-V/ I-F-D-P-X-K-R-I-I-V-D-E-A-L (group B K-M-L-I-F-D-P-S/ T-K-R-I-S-V-T-E-A-L (group C) and L-L-E-R/K-L-L-A-FD-P-K-D-R-P-T-A-E-E-A-L (group D) (Table 4).

\section{Phylogeny}

An unrooted phylogenetic tree was constructed to infer group specific relationships of MAPKs. Upon phylogenetic analysis, all studied MAPK genes are fell into six different clusters, that are named according to the MAPK grouping of $A$. thaliana. In A. thaliana, MAPK genes are classified into four different groups (A, B, C, and $\mathrm{D})$ based on their evolutionary relationship and

\begin{tabular}{|c|c|c|c|}
\hline \multicolumn{4}{|c|}{ Conserved signature motifs of MAPKs } \\
\hline $\begin{array}{l}\text { N-Terminal } \\
\text { conserved } \\
\text { motifs }\end{array}$ & $\begin{array}{l}\text { Some other conserved } \\
\text { Motif Present in } \\
\text { N-terminal End }\end{array}$ & $\begin{array}{l}\text { Activation loop } \\
\text { motifs }\end{array}$ & $\begin{array}{l}\text { C-Terminal } \\
\text { motifs }\end{array}$ \\
\hline Group D & Group A: A-K-Y, N-K-Y & $\begin{array}{l}\text { Group A: T-E-Y, } \\
\text { T-Q-Y }\end{array}$ & $\begin{array}{l}\text { Group A \& B: } \\
\text { S-D-Y, S-E-Y, } \\
\text { T-D-Y }\end{array}$ \\
\hline S-D-Y, S-E-Y & Group B: S-K-Y, R-K-Y & $\begin{array}{l}\text { Group B: T-E-Y, } \\
\text { M-E-Y, T-E-C, T- } \\
\text { V-Y }\end{array}$ & $\begin{array}{l}\text { Group C: D-N- } \\
\text { Y, S-Q-Y }\end{array}$ \\
\hline \multirow[t]{3}{*}{ T-D-Y, T-E-Y } & Group C: T-K-Y & Group C: T-E-Y & $\begin{array}{l}\text { Group D: S-K-Y } \\
\text { T-K-Y, S-R-Y, } \\
\text { S-N-Y }\end{array}$ \\
\hline & $\begin{array}{l}\text { Group D: S-Q-Y, N-R-Y, } \\
\text { S-R-Y }\end{array}$ & Group D: T-D-Y & \\
\hline & & $\begin{array}{l}\text { Group E: T-S-Y, } \\
\text { T-E-M, T-Q-M, } \\
\text { T-R-M }\end{array}$ & \\
\hline
\end{tabular}

presence of the T-D-Y and T-E-Y phosphorylation motif. In this study, MAPKs are categorized into six different groups namely group A (red), B (blue), C (pink), D (purple), E (teal) and F (green) (Figure 5, Additional file 5). Two new group of MAPK (group E and F) are generated during this analysis. The new group $\mathrm{E}$ and $\mathrm{F}$ are mainly shared by MAPKs of lower eukaryotic and gymnosperm plants such as CsubMPK7, MpMPK13, SmMPK10, CreinMPK7, VcMPK5, CsubMPK3, PaMPK10, PaMPK7-2, PaMPK5, PaMPK14, CreinMPK4-1, VcMPK4-1, OIMPK6, MpMPK4. The phylogenetic analysis revealed that 89,128 , 100, 258, 10 and 4. MAPKs fall into group A, B, C, D, E, and F respectively (Additional file 5, Table 5). The average overall phylogenetic mean distance of plant MAPK is 0.54 (standard error 0.029). During phylogenetic distance estimation, all the positions with less than 95\% site coverage are eliminated. That is, fewer than $5 \%$ alignment gaps. The missing data and ambiguous bases are allowed at any position.

\section{Statistical analysis}

Different statistical analysis was carried out to infer the statistical significance of the study. In Tajima's relative rate test, different MAPK sequences were taken randomly from active data as different groups. Analysis was repeated for two times by taking random MAPK sequences into different taxonomic group A, B and C (these groups are statistical groups and should not be confused with MAPK groups). More specifically, group $\mathrm{C}$ was used as out group. When we took MgMPK4-1 (group A), GmMPK16-3 (group B) and AtPIN1 (group C), resulted $p$-value was 0.05935 and $X^{2}$-test result was 3.56 (Table 6). When MgMPK4-1, GmMPK16-3 and AtCBL1 are taken as group $\mathrm{A}, \mathrm{B}$ and $\mathrm{C}$ respectively, the $p$-value result was 0.00468 and $X^{2}$-test result was 8.00 . In both the cases statistical value was found to be significant (Table 6). The $P$-value less than 0.05 is often used to reject the null hypothesis of equal rates between lineages $(\mathrm{p} \leq 0.01$ : very strong presumption against null hypothesis, $0.01<\mathrm{p} \leq 0.05$ strong presumption against null hypothesis, $0.05<\mathrm{p} \leq 0.1$ low presumption against null hypothesis, $\mathrm{p}>0.1$ no presumption against null hypothesis). The analysis involved 3 amino acid sequences. All positions containing gaps and missing data were eliminated. In Tajima's test 


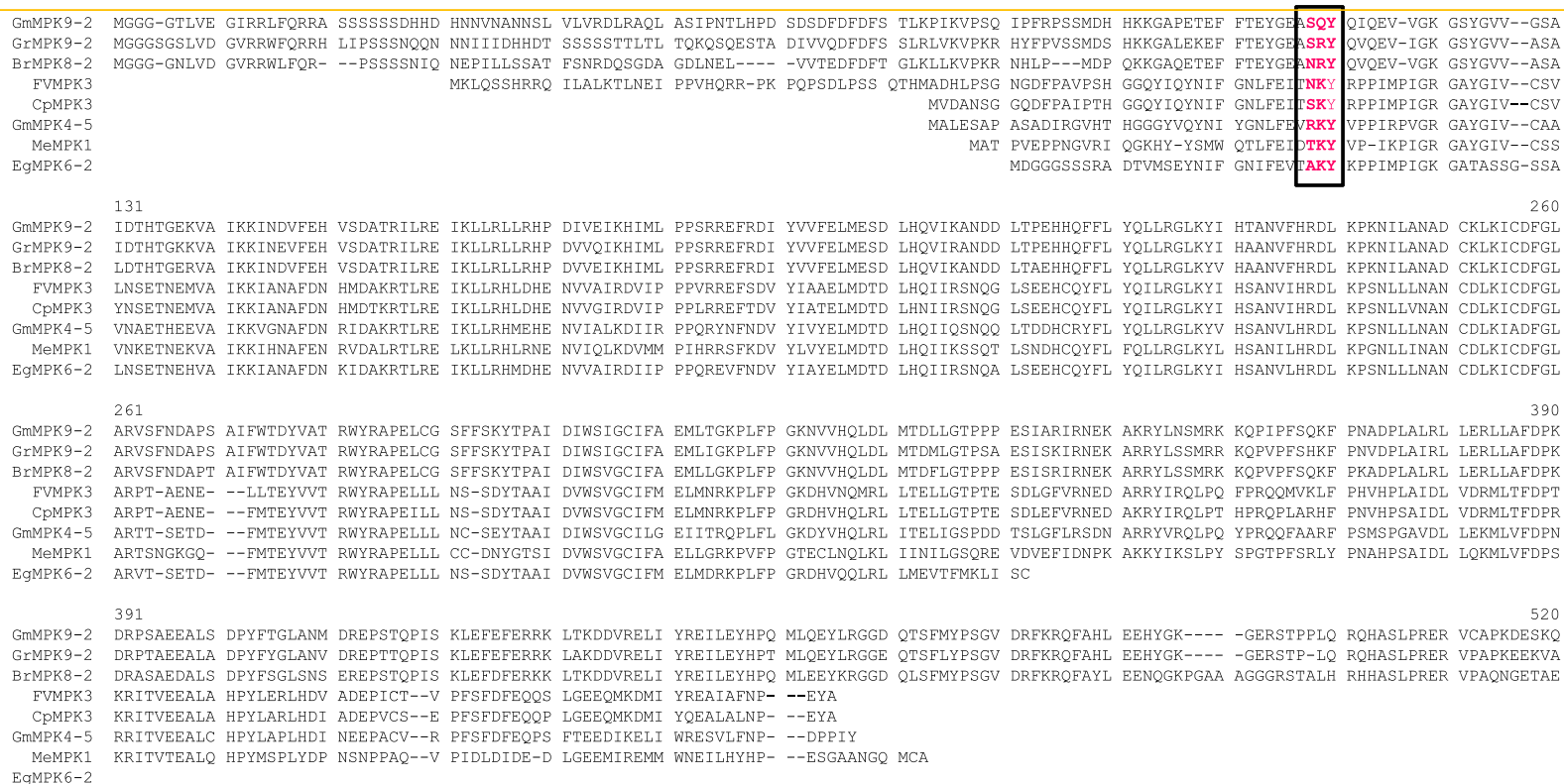

Figure 2 The $\mathbf{N}$-terminal conserved motifs of plant MAPKs. These motifs are immediately followed by presence of N-terminal SEY, SDY, TEY, TDY motifs of group D MAPKs. This picture is a pictorial representation of different motifs taken from different groups of MAPKs. For details, please see Table 2 and Additional file 4.

statistics (D) for neutrality, the D value was found to be 4.904140 ( $\mathrm{D}=4.904140)$ (Table 7). All the positions with less than $95 \%$ site coverage are eliminated during Tajima's test for neutrality. There were a total of 322 positions in the final dataset.

\section{Gene duplication}

Chromosomes are evolved via fusion, fission, insertion, and duplication events, allowing evolution of chromosome size and number, and hence the genes. Gene duplication is the major force acting on the evolution of different species, and the gene families are groups of

Table 3 Table presenting different conserved consensus sequences present in plant MAPKs

\begin{tabular}{|c|c|}
\hline \multicolumn{2}{|c|}{ Common conserved signature sequences of MAPKs } \\
\hline $\begin{array}{l}\mathrm{N}-\text { Terminal conserved } \\
\text { consensus sequences }\end{array}$ & $\begin{array}{l}\text { C-Terminal conserved } \\
\text { consensus sequences }\end{array}$ \\
\hline I-G-X-G-x-Y-G-x-V & T-R-W-Y-R-A-P-E-L \\
\hline $\mathrm{I}-\mathrm{K}-\mathrm{K}-\mathrm{I}-\mathrm{X}_{3}-\mathrm{F}$ & I-D-x-W-S-I/N-G-C \\
\hline$D-A-x-R-x-L-R-E$ & Q-X-L-L-X-F-D-P \\
\hline \multicolumn{2}{|l|}{ F-X-D-I-Y-X $-E-L-M$} \\
\hline \multicolumn{2}{|l|}{$D-L-X_{2}-V-I$} \\
\hline \multicolumn{2}{|l|}{$D-X-L-X_{2}-E-H$} \\
\hline \multicolumn{2}{|l|}{ Q-X-L-R-X-L-K-Y-X-H } \\
\hline \multicolumn{2}{|l|}{ H-R-D-L-K-P-X-N } \\
\hline L-X-N-X-N-C-X-L-K-I-X-D-F-G-L-A-R & \\
\hline
\end{tabular}

genes generated by duplication. The sizes of gene families reflect the number of duplicated genes, which are known as paralogs. Several plant MAPKs analyzed during this study were found to be duplicate genes resulting in several paralogous genes (Additional file 1). The plants with duplicated genomes give rise to more duplicated MAPKs relative to species with non duplicated genomes. Accordingly, species such as G. max, G. raimondii, and $M$. domestica contain more duplicated genes. Nevertheless, almost all species posses duplicated MAPKs in their genome. The gene duplication result of plant MAPKs those contained novel activation loop motif are reported in table (Table 8). All the MAPK resulted in $\mathrm{z}$-score above four with $100 \%$ level of confidence.

\section{MAPK groups MAPKs in monocotyledonous plants}

Among the 40 different plant species analyzed during this study, six were monocotyledonous plants (B. distachyon, O. sativa, $P$. virgatum, S. italica, S. bicolour and Z. mays) (Table 1). Our study revealed that $O$. sativa contains 17 MAPKs, not 15 as reported earlier. Among monocot species, $P$. virgatum contains the highest number of MAPKs in its genome (27).

\section{MAPKs in dicotyledonous plants}

The MAPK gene family of dicotyledonous plants has shown large variations among MAPK gene family 


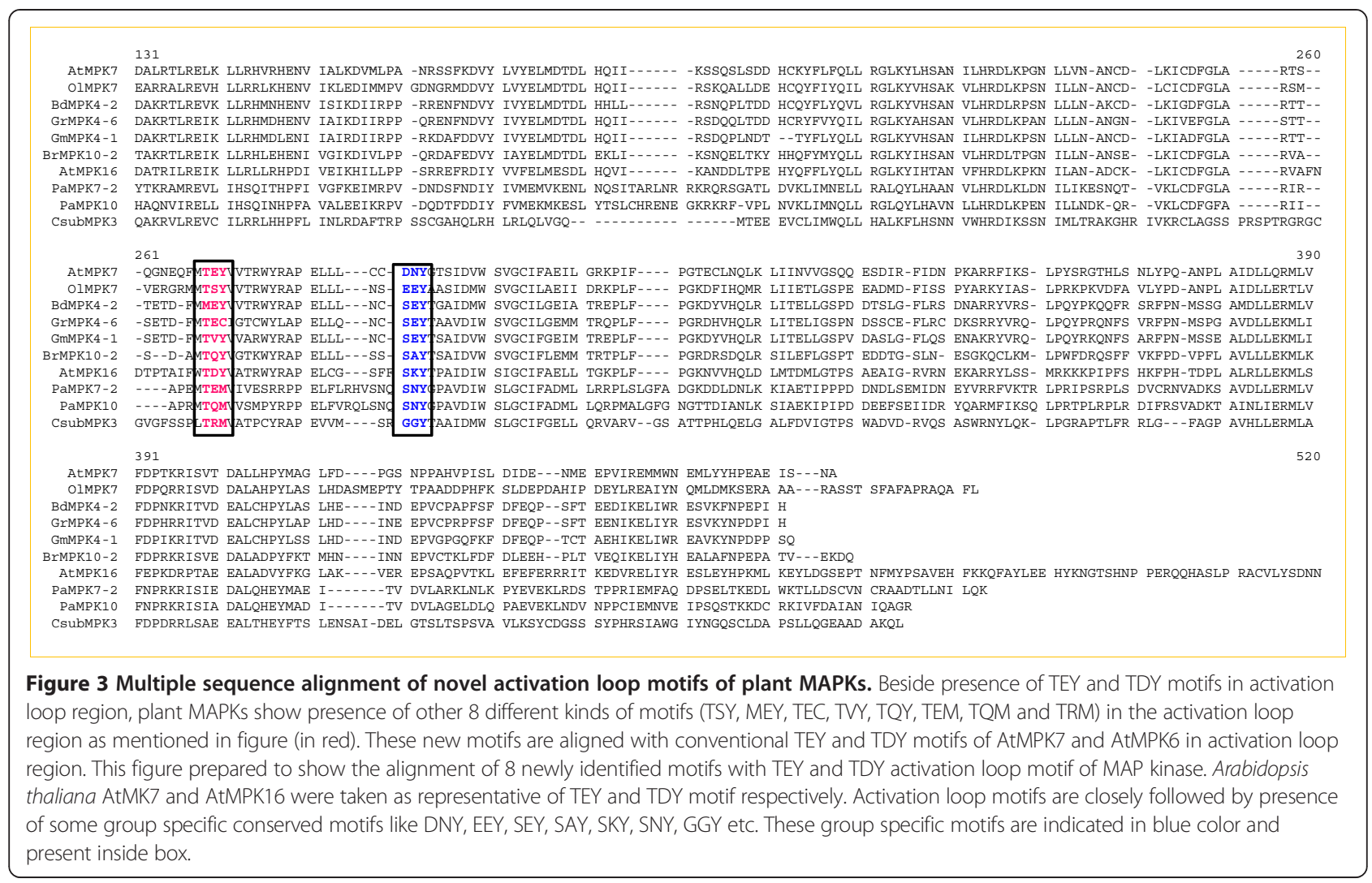

members (Table 1). Among 40 different species investigated herein, 26 were dicot plants. This group contained as few as 6 MAPK gene in M. guttatus to as many as 31 in soybean (G. max). Investigation of MAPKs in G. $\max$ in another study using the HMM (hidden Markov model) approach also showed 35 MAPKs; however, four of them are indeed 'MAPK-likes' genes, making the actual MAPK number 31 .

\section{MAPKs in lower photosynthetic eukaryotes (Algae, Moss and Pteridophyte)}

The lower photosynthetic groups includes four algae, one bryophyte, one pteridophyte and one gymnosperm species (Table 1). Multiple MAPKs were seen in several species of algae (Table 1$)$. Our study also showed multiple MAPKs (TEY, 2/3/4 and TDY, 1/2) in unicellular and multi-cellular algae (Table 5). A genome survey of $S$. moellendorffii, a model lycophyte (non-seed vascular plant) and a primitive species revealed the presence of six MAPKs with single MAPKs in group A and B, whereas two MAPKs were present in group $C$ and D. As a result, there are four MAPKs with the TEY motif and only two with the TDY motif. Among the mosses and algae, $P$. patens and O. lucimarinus lacks 'group A' type MAPKs, while $V$. cartieri lacks 'group B' and 'group C' type MAPKs and C. reinhardtii and C. subellipsoidea do not possess 'group C' MAPKs. Interestingly, none of the studied lower photosynthetic eukaryotes or land plants (both mono and dicot species) lacked 'group D' type MAPKs.

\section{Discussion}

Nomenclature and identification of MAPKs

It is very important to assign an appropriate and specific name to each member of the family to enable a thorough understanding of it. Therefore, we provided unique names to all 589 identified MAPKs across the plant lineage using the orthologous based nomenclature system proposed by Hamel et al. [21]. In the traditional naming system, names are assigned to gene(s) that are identified and cloned first, regardless of their similarities to other gene(s). For example, if someone cloned the ThMPK gene from Thelluginella halophila first, it named as ThMPK1, regardless of its orthologous similarity with other MPKs. Accordingly, if this ThMPK1 has orthologous similarity with AtMPK6, it should be named ThMPK6, but this does not happen. However, orthology lends the legitimacy to transfer of functional similarities from its ancestors [22-24]. As a result, orthology based nomenclature can provide succinct information regarding its orthologous counterpart gene. Practically, it is difficult to study every individual MAPK gene in all plant species to understand their specific roles in different 

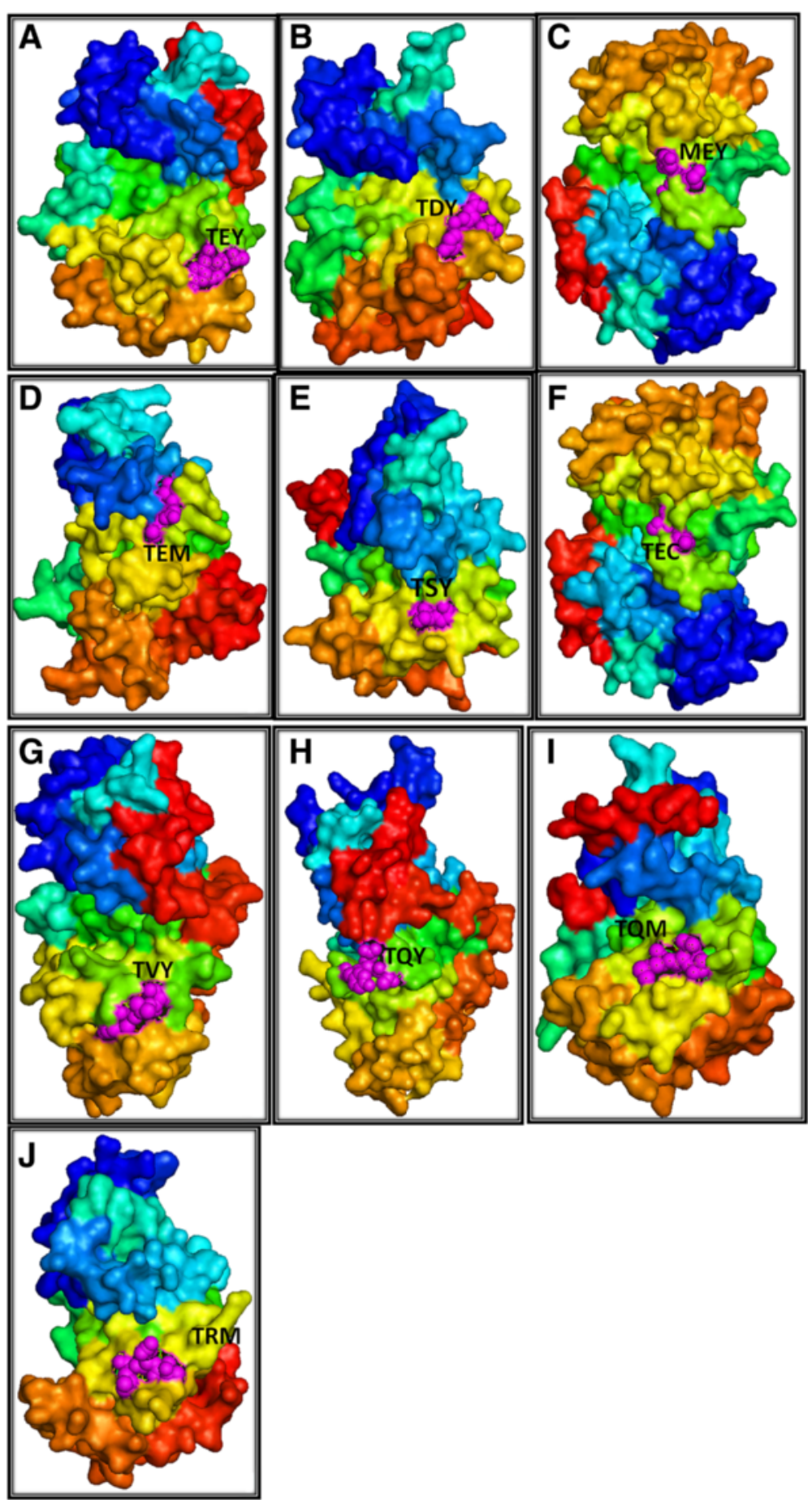

Figure 4 Molecular structure of plant MAPKs showing their activation loop motif. The structures were modeled by homology based modeling procedure using the Phyre2 server. The activation loop motifs were labeled using Pymol. Figures A, B, C, D, E, F, G, H, I, J represents T-E-Y (AtMPK1), T-D-Y (AtMPK20), M-E-Y (OsMPK16-2), T-E-M (PaMPK7-2), T-S-Y (OIMPK7), T-E-C (GrMPK4-6), T-V-Y (GmMPK4-1), T-Q-Y (BrMPK10-2), T-Q-M (PaMPK10) and T-R-M (CsubMPK3) motif respectively. 
Table 4 Table showing predicted group specific common docking (CD) sites of plant MAPKs

\begin{tabular}{ll}
\hline Predicted common docking (CD) sites of MAPKs \\
\hline Group A & K-M-L-T-F-D-P-K/R-Q/K-R-I-T-V-E-D/E-A-L \\
Group B & K-M-L-V/I-F-D-P-X-K-R-II-V-D-E-A-L \\
Group C & K-M-L-I-F-D-P-S/T-K-R-I-S-V-T-E-A-L \\
Group D & L-L-E-R/K-L-L-A-F-D-P-K-D-R-P-T-A-E-E-A-L \\
\hline
\end{tabular}

aspects of plant biology. As orthology lends the legitimacy of common ancestry and evolutionary function, orthology based nomenclature will provide ideas regarding possible roles of specific genes in the plant species being investigated. This system of nomenclature can be further extended to newly identified gene families of other plant species.

To date, MAPK gene family members of only few plant species have been reported, including Oryza sativa [14], Arabidopsis thaliana [13], Zea mays [15], Brachypodium distachyon [18], Canola (Brassica napus) [16] and Malus domestica [17]. Although MAPKs from these plant species are previously identified, we have included them here to broaden the study. Inclusion of these species in our study led to identification of some new members of MAPKs. An earlier study by Hamel et al., revealed that the O. sativa genome contains 16 members of the MAPK gene family [21]. However, we recently identified 17 MAPK gene family members from $O$. sativa. Additionally, Zhang et al., reported 26 members of the MAPK gene family from Malus domestica [17], but we found that $M$. domestica contains 28 members.

\section{Genomics of MAPKs}

All species are confined by specific numbers of fundamental traits known as chromosome [25,26]. The number of sets of chromosomes varies among genera and species, as well as within species. Some species are functionally haploid (e.g., Chlamydomonas, Volvox, Coccomyxa, Ostreococcus, Physcomitrella, Selaginella), or diploid (Oryza sativa, Arabidopsis thaliana, Gossipium raimondii, Glycine max, Zea maize) (Table 1). The genome size of a specific species is directly correlated with the ploidy level (haploid, diploid or polyploidy) of the organism [27,28]. The lower eukaryotic organisms such as Chlamydomonas, Volvox, Coccomyxa, Ostreococcus, Physcomitrella, and Selaginella are very simple life forms relative to higher eukaryotic angiosperms; therefore, they encode very few MAPK genes relative to higher plants. For example, the lower eukaryotic algae $O$. lucimarinus encodes only three MAPKs, while $C$. subellipsoidea encodes only four. C. reinhardtii and $S$. moellendorffii encode six MAPKs each, whereas $P$. patens encodes eight. The plants G. max has tetraploid genome; hence, its genome size is larger than those of O. sativa and A. thaliana. Owing to the ploidy nature of the genome, organisms encode more MAPKs [27,29,30]. G. max encodes maximum of 31 MAPKs, whereas $B$. rapa encodes for 30 MAPKs. Similarly, M. domestica and G. raimondii encodes for 28 MAPKs each. The presence of higher numbers of MAPK genes in these plants is attributed to their bigger genome size and ploidy level or whole genome duplication. The number of MAPK genes within each family varies from species to species depending upon the complexity and ploidy level of plants (Table 1).

During this study, we found that MAPK genes harbored several introns (Additional file 1). Additionally, different genes contained different numbers of introns ranging from zero (intronless) to fourteen. We did not find any group specific conserved intron organization for groups $\mathrm{A}, \mathrm{B}, \mathrm{C}$, and E MAPK genes. However, group D MAPKs harbored seven to fourteen introns. It has been reported that intron organization is conserved at levels up to $10-20 \%$ between orthologs $[31,32]$, and the presence of 126 MAPK genes containing nine introns in their gene constitutes $21.39 \%$ orthology. Accordingly, our findings are in agreement with earlier findings regarding the orthology based evolution of introns in plants. These findings confirm that group D MAPKs are evolutionarily more conserved than other groups of MAPKs.

\section{Conserved motifs and domains}

Mitogen activated protein kinases are multigene families characterized by the presence of an activation loop T-E-Y/ T-D-Y motif. [6,21] These conserved motifs are target phosphorylation sites of upstream MAPKK (mitogen activated protein kinase kinase).To date, there have been no reports regarding the presence of $\mathrm{N}$-terminal conserved motifs in MAPKs. We found that group D MAPKs contains conserved N-terminal T-E-Y/T-D-Y/S-D-Y and S-E-Y motifs (Figure 1A, 1B, Table 2). Chlamydomonas and Volvox share a common A-V-H motif instead of the S-EY/S-D-Y/T-E-Y and T-D-Y motif. Protein phosphorylation can occur on multiple distinct sites throughput the given protein $[33,34]$. Hence the presences of N-terminal conserved motifs are may be target phosphorylation sites of some other unknown kinases. In addition to the presence of these conserved motifs, the $\mathrm{N}$-terminal region of MAPKs also contains several other conserved consensus amino acids, I-G-x-G-x-Y-G-x-V, I-K-K-I- $x_{3}-F$, D-A-x-R-xL-R-E, F-x-D-I-Y- $x_{3}-E-L-M, \quad D-L-x_{2}-V-I, \quad D-x-L-x_{2}-E-H$, Q-x-L-R-x-L-K-Y-x-H, H-R-D-L-K-P-x-N, and L-x-L-x-NC-x-L-K-I-x-D-F-G-L-A-R (Figure 1A, Table 3). These conserved consensus amino acid sequences may be considered as signature consensus of MAPKs. The presence of conserved amino acids consensuses in MAPKs reflects their common evolutionary ancestry. Earlier, Hanks (2003) reported that the protein kinase super-family contains the G-x-G-x-x-G conserved consensus sequence [4], which is similar to the results of our study, in which I-G-x-G-x-Y- 


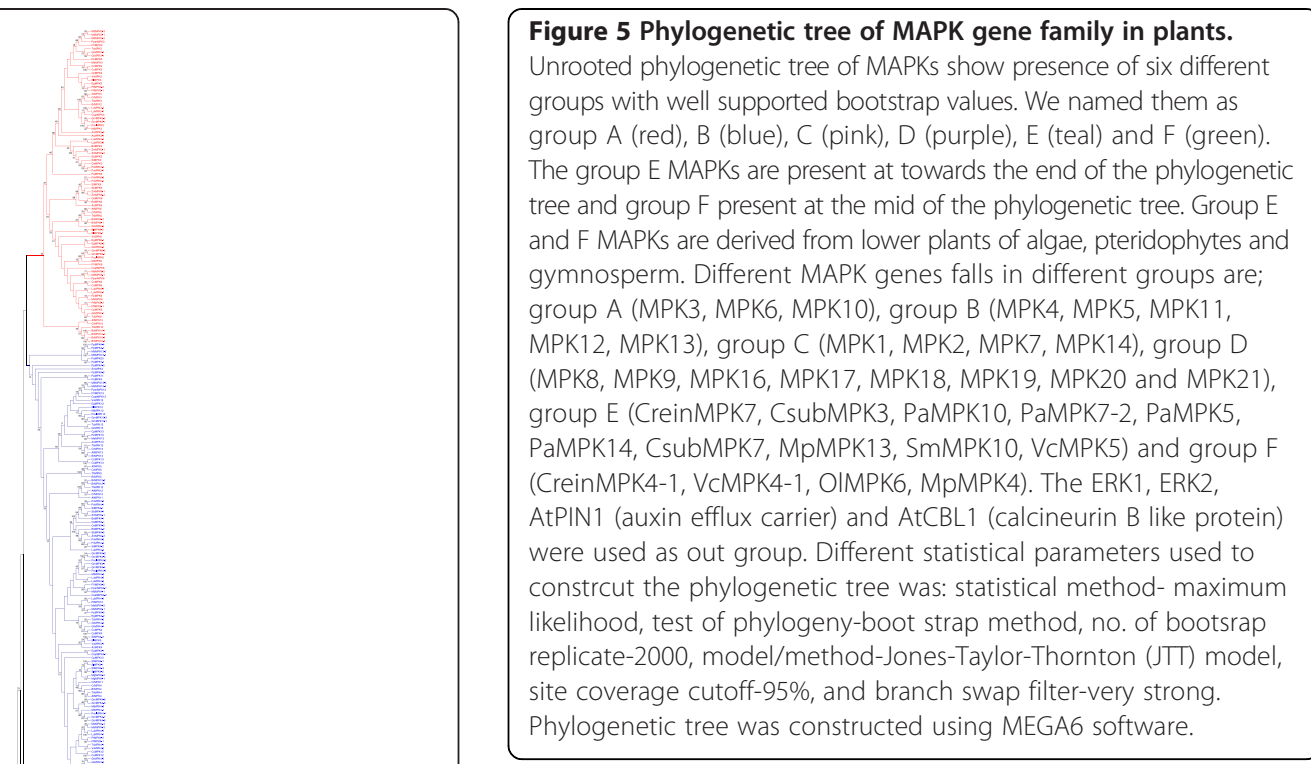

G-x- $\mathrm{V}$ was conserved instead of $\mathrm{G}-\mathrm{x}-\mathrm{G}-\mathrm{x}-\mathrm{x}-\mathrm{G}$ across all MAPKs (Figure 1A, Table 3). In addition to the T-E-Y/T-D$Y$ activation loop motif of MAPK, several new and novel activation loop motifs were identified those includes M-E-Y (group B), T-E-M (group D), T-S-Y (group D), T-E-C (group B), T-V-Y (group B), T-Q-Y (group A), T-Q-M (group D) and T-R-M (group D) (Table 2). None of the identified motifs belonged to group C MAPK. These new motifs are assumed to be undergoing recent evolution to expand the diversity of MAPKs; however, the absence of a new kind of activation loop motif for group C MAPK indicates that this group is more conserved than other groups of MAPKs. The presence of diverse activation loop motifs are may be for target phosphorylation sites for diverse kinases that can facilitate phosphorylation events more easily to overcome selective pressure. These activation loop conserved motifs are closely followed by several other group specific conserved motifs, including S-D-Y, S-E-Y, T-D-Y (group A and B MAPKs), D-N-Y, S-Q-Y (group C MAPKs), S-K-Y, and T-K-Y (group D MAPKs) (Table 2). These motifs are may be putative phosphorylation sites of other kinases. The presence of the $\mathrm{N}$-terminal conserved activation loop motif immediately followed by the presence of another conserved motif greatly reflects presence of wide arrays of phosphorylation sites of MAPKs, which may be target phosphorylation sites of different other kinases. The presence of N-terminal S-D-Y/S-E-Y/T-D-Y motifs along with C-terminal S-D-Y/S-E-Y/T-D-Y/S-Q-Y/D-N-Y/S-R-Y/S-KY/T-K-Y/A-K-Y/S-N-Y/G-R-Y motifs reflects challenges to defining MAPKs; however, these MAPKs can now be better defined as group specific. Although it has been proposed that the T-E-Y and T-D-Y motifs at the activation loop are important for activation of MAPKs, the presence of $\mathrm{N}$-terminal and C-terminal S-D-Y/S-E-Y/T-D-Y as well 
Table 5 Table showing group specific distribution of different MAPKs in plants

\begin{tabular}{|c|c|c|c|c|c|c|c|}
\hline \multirow{3}{*}{$\begin{array}{l}\text { Sl. } \\
\text { No. }\end{array}$} & \multirow[t]{3}{*}{ Name of plant species } & \multicolumn{6}{|c|}{ Mitogen activated protein kinase } \\
\hline & & \multicolumn{3}{|l|}{$\overline{T E Y}$} & \multirow{2}{*}{$\begin{array}{l}\text { TDY } \\
\text { Group D }\end{array}$} & \multirow[t]{2}{*}{ Other group } & \multirow[t]{2}{*}{ Tota } \\
\hline & & Group A & Group B & Group C & & & \\
\hline 1 & Aquilegia coerulea & 3 & 2 & 3 & 2 & & 10 \\
\hline 2 & Arabidopsis thaliana & 3 & 5 & 4 & 8 & & 20 \\
\hline 3 & Brachipodium distachyon & 2 & 2 & 3 & 9 & & 16 \\
\hline 4 & Brassica rapa & 7 & 5 & 4 & 14 & & 30 \\
\hline 5 & Capsella rubella & 3 & 5 & 2 & 8 & & 18 \\
\hline 6 & Carica papaya & 2 & 2 & 1 & 4 & & 9 \\
\hline 7 & Chlamydomonas reinhardtii & 0 & 0 & 2 & 2 & 2 & 6 \\
\hline 8 & Citrus clememtina & 2 & 3 & 2 & 5 & & 12 \\
\hline 9 & Citrus sinensis & 2 & 3 & 2 & 5 & & 12 \\
\hline 10 & Coccomyxa subellipsoidea & 0 & 0 & 1 & 1 & 2 & 4 \\
\hline 11 & Cucumis sativus & 2 & 3 & 2 & 7 & & 14 \\
\hline 12 & Eucalyptus grandis & 3 & 3 & 1 & 6 & & 13 \\
\hline 13 & Fragaria vesca & 2 & 3 & 2 & 4 & & 11 \\
\hline 14 & Glycine max & 4 & 10 & 4 & 13 & & 31 \\
\hline 15 & Gossipium raimondi & 5 & 7 & 6 & 10 & & 28 \\
\hline 16 & Linum usitatissimum & 6 & 6 & 4 & 8 & & 24 \\
\hline 17 & Malus domestica & 5 & 8 & 5 & 10 & & 28 \\
\hline 18 & Manihot esculenta & 2 & 5 & 2 & 8 & & 17 \\
\hline 19 & Medicago truncatula & 2 & 4 & 5 & 6 & & 17 \\
\hline 20 & Micromonas pusila & 0 & 0 & 1 & 1 & 2 & 4 \\
\hline 21 & Mimulus guttatus & 0 & 2 & 1 & 3 & & 6 \\
\hline 22 & Oryza sativa & 2 & 2 & 2 & 10 & 1 & 17 \\
\hline 23 & Ostreococcus lucimarinus & 0 & 1 & 1 & 1 & & 3 \\
\hline 24 & Panicum virgatum & 4 & 4 & 4 & 15 & & 27 \\
\hline 25 & Phaseolus vulgaris & 2 & 4 & 2 & 6 & & 14 \\
\hline 26 & Physcomitrella patens & 0 & 4 & 2 & 2 & & 8 \\
\hline 27 & Picea abies & 1 & 2 & 5 & 2 & 4 & 14 \\
\hline 28 & Populus trichocarpa & 4 & 3 & 4 & 10 & & 21 \\
\hline 29 & Prunus persica & 2 & 3 & 2 & 5 & & 12 \\
\hline 30 & Ricinus communis & 2 & 3 & 2 & 5 & & 12 \\
\hline 31 & Selaginella moellendorffii & 0 & 1 & 2 & 2 & 1 & 6 \\
\hline 32 & Setaria italica & 2 & 2 & 2 & 10 & & 16 \\
\hline 33 & Solanum lycopersicum & 3 & 4 & 2 & 8 & & 17 \\
\hline 34 & Solanum tuberosum & 0 & 3 & 2 & 7 & & 12 \\
\hline 35 & Sorghum bicolor V 1.4 & 2 & 2 & 2 & 10 & & 16 \\
\hline 36 & Thellungiella halophila & 3 & 4 & 2 & 7 & & 16 \\
\hline 37 & Theobroma cacao & 2 & 3 & 2 & 5 & & 12 \\
\hline 38 & Vitis venifera & 2 & 3 & 2 & 5 & & 12 \\
\hline 39 & Volvox carteri & 0 & 0 & 1 & 2 & 2 & 5 \\
\hline 40 & Zea mays & 4 & 2 & 2 & 11 & & 19 \\
\hline
\end{tabular}


Table 6 Tajima's relative rate test

\begin{tabular}{|c|c|c|}
\hline \multirow[t]{2}{*}{ Configuration } & \multicolumn{2}{|l|}{ Count } \\
\hline & $\begin{array}{l}\text { MgMPK4-1, } \\
\text { GmMPK16-3 AtPIN1 }\end{array}$ & $\begin{array}{l}\text { MgMPK4-1, } \\
\text { GmMPK16-3 AtCBL1 }\end{array}$ \\
\hline $\begin{array}{l}\text { Identical sites in all } \\
\text { three sequences }\end{array}$ & 15 & 7 \\
\hline $\begin{array}{l}\text { Divergent sites in all } \\
\text { three sequences }\end{array}$ & 64 & 44 \\
\hline $\begin{array}{l}\text { Unique differences in } \\
\text { sequence } A\end{array}$ & 13 & 8 \\
\hline $\begin{array}{l}\text { Unique differences in } \\
\text { sequence B }\end{array}$ & 5 & 0 \\
\hline $\begin{array}{l}\text { Unique differences in } \\
\text { sequence } C\end{array}$ & 70 & 47 \\
\hline$P$-value & 0.05935 & 0.00468 \\
\hline$x^{2}$-test & 3.56 & 8.00 \\
\hline
\end{tabular}

Tajima's relative rate test was carried out by randomly comparing three phylogenetically distant sequences in each case by distributing them into three distinct group. In column 2, group A (OsMPK6), B (PaMPK8) and C (SmMPK16-2); column 3, group A (AtMPK1), B (PaMPK8) and C (CreinMPK4-2); column 4, group A (SiMPK14), B (VcMPK9) and C (PaMPK3); column 5, group A (SbMPK14), B (PpMPK4-3), and C ( PaMPK3). Test was replicated for four times. In all the four cases, statistical result was found to be significant.

as other motifs in close vicinity to the activation loop indicates that more in depth investigations are needed to confirm their functions. The presence of group specific conserved motifs and domains explains the orthology based evolution of MAPKs from the common ancestors.

In fungi, MAPKs regulates five different pathways namely CWI (cell wall integrity), HOG (high osmolarity glycerol), Kss, Fus, and Smk1 (sporulation and meiosis) [35]. The CWI pathway is carried out by fungal Mpk1 that contain T-E-Y motif in its activation loop domain, HOG pathway is carried out by Hog1 that contain T-G$\mathrm{Y}$ motif, Kss pathway is responsible for filamentous growth in fungi and Kss1 contain T-E-Y motif, Fus pathway is responsible for mating and cell cycle arrest and contain T-E-Y motif in its activation loop domain. The fungal Smk1 pathway responsible for meiosis and sporulation contain T-N-Y motif in its activation loop domain. From these report, it is evident that MAPK that contain different activation loop motif controls different pathways. Presence of several new activation loop motifs in plants may be responsible for some novel pathways which are yet to be elucidated.

Table 7 Tajima's test for neutrality

\begin{tabular}{llllll}
\hline $\boldsymbol{m}$ & $\boldsymbol{S}$ & $\boldsymbol{p}_{\mathbf{s}}$ & $\boldsymbol{\theta}$ & $\boldsymbol{\pi}$ & $\boldsymbol{D}$ \\
\hline 594 & 320 & 0.993789 & 0.142719 & 0.377879 & 4.904140 \\
\hline
\end{tabular}

The analysis involved 589 amino acid sequences. All positions with less than $95 \%$ site coverage were eliminated. That is, fewer than $5 \%$ alignment gaps, missing data, and ambiguous bases were allowed at any position. There were a total of 325 positions in the final dataset. Evolutionary analyses were conducted in MEGA6. Abbreviations: $m=$ number of sequences, $n=$ total number of sites, $S=$ Number of segregating sites, $p_{\mathrm{s}}=S / n, \Theta=p_{\mathrm{s}} / \mathrm{a}_{1}, \pi=$ nucleotide diversity, and $D$ is the Tajima test statistic.
Table 8 Gene duplication analysis of some selective plant MAPKs that contain novel activation loop motif

\begin{tabular}{llll}
\hline $\begin{array}{l}\text { Gene } \\
\text { name }\end{array}$ & $\begin{array}{l}\text { Representative } \\
\text { motif }\end{array}$ & Z-score & Level of confidence (\%) \\
\hline AtMPK1 & T-E-Y & 19.46 & 100 \\
AtMPK20 & T-D-Y & 11.88 & 100 \\
OsMPK16-2 & M-E-Y & 6.85 & 100 \\
PaMPK7-2 & T-E-M & 7.92 & 100 \\
OIMPK7 & T-S-Y & 8.48 & 100 \\
GrMPK4-6 & T-E-C & 13.94 & 100 \\
GmMPK & T-V-Y & 13.67 & 100 \\
BrMPK & T-Q-Y & 7.91 & 100 \\
PaMPK10 & T-Q-M & 13.41 & 100 \\
CsubMPK3 & T-R-M & 7.75 & 100 \\
\hline
\end{tabular}

Analysis was carried out by Pinda (pipeline for intraspecies duplication analysis). Analysis shows all the MAPKs that contain novel activation loop motif resulted in z-score above four. The Z-score above four is considered highly significant to be duplicated.

\section{Common docking site}

It was previously assumed that substrate specificity of an enzyme is determined by stereo-chemical complementarities with its active site [36-39]; however, these preferences are not stringent enough. It has since been found that, in addition to substrate target site preferences, many protein kinases uses dedicated modular protein-protein interaction docking domains, interactions involving binding of the surface of the catalytic domain, but distinct from catalytic active sites [40-42]. These docking grooves are bind to the short peptide docking motifs that are separated from the substrate motif. Although these two supplemental recognition strategies are not mutually exclusive, development of these alternative modes of recognition provides a very simple method to meet increasing evolutionary requirements [41]. Protein kinases often recognize their substrates and regulators through docking interaction sites that occurs outside the active site $[43,44]$, and these interactions can help us to understand the kinase networks. The MAPKs contains group specific conserved docking domains, K-M-L-T-FD-P-K/R-Q/K-R-I-T-V-E-D/E-A-L (group A), K-M-L-V/ I-F-D-P-x-K-R-I-I-V-D-E-A-L (group B), K-M-L-I-F-D-PS/T-K-R-I-S-V-T-E-A-L (group C) and L-L-E-R/K-L-L-AF-D-P-K-D-R-P-T-A-E-E-A-L (group D) (Table 4). The presence of group specific docking domains in MAPKs suggests that different MAPKs targets are group specific. Similarly, the presence of group specific conserved docking domains suggests, evolution of MAPKs are orthologous based and are group specific.

\section{Phylogeny of MAPKs}

To understand the evolutionary expansion of MAPKs belonging to 40 different plant species, an unrooted tree was constructed from alignments of their full length protein 
sequences. The analysis revealed that all MAPKs fell into six different groups with well supported bootstrap values. We named them as group A (red), B (blue), C (pink), D (purple), E (teal) and F (green) (Figure 5, Additional file 5). The clustering of MAPKs into different groups reflects their orthology based origin from common ancestors. The cluster of group E MAPKs belonged to lower eukaryotic organisms and fell in the distal end (basal part) of the phylogenetic tree (Figure 5, Additional file 5). The finding of group E MAPKs from lower green algae and other organisms fell as an independent group suggests that these MAPKs were evolved independently and diverged during evolution to higher eukaryotes. Independent grouping of group E and F MAPKs may also indicate lower eukaryotic specific functions and their independent evolution [45-47]. Although biological functions of all MAPKs are not yet understood, MAPKs of the same subgroup are likely to be involved in similar physiological responses, and hence similar functions. The presence of lower eukaryotic specific group F MAPKs in the middle of the phylogenetic tree may also reflects their roles in evolution of MAPKs by subsequent divergence and duplication. During speciation events, these MAPKs became diversified and evolved as multigene families from common ancestors. It has been reported that multiple members of specific gene families of a particular organism are the natural products generated from the evolutionary history experienced by an organism [48-51]. Accordingly, the presence of several members in a gene family reflects the succession of genomic rearrangement, and its expansion is due to extensive duplication and diversification that occurs during the course of evolution $[52,53]$. Expansion of the gene family may be involved in different developmental processes of plants [54-58].

\section{Statistical analysis}

In the random sampling of MAPK sequences in Tajima's relative rate test, we found significant $p$-value and $X^{2}$ test result in both the studied groups. This implies that our study is statistically significant (Table 6). In Tajima's test for neutrality, the Tajima's D test result is 4.904140 $(\mathrm{D}=4.904140)$ (Table 7). In Tajima's $\mathrm{D}$ test, when $\mathrm{D}=0$, the average heterozygosity became equal to number of segregating sites and can be interpreted as expected variation is similar to observed variation $[59,60]$. The evolving population can be due to mutation-drift equilibrium and no evidence of selection. When $\mathrm{D}<0$, the average heterozygosity is lower than number of segregating sites $[59,60]$. In this case, it can be interpreted as rare alleles are present at very low frequencies and recent selective sweep led to population expansion after recent bottleneck that contain the linkage to swept a gene. When $\mathrm{D}>0$, the average heterozygosity is more than that of segregating sites and can be considered as presence of multiple alleles; some at low and others at high frequencies $[59,60]$. This creates balancing in selection by sudden contraction in population. Tajima's negative $\mathrm{D}$ value signifies excess low frequency of polymorphism relative to expectation. This indicates expansion in a population size by selective sweep or by purifying selection. Tajima's positive $\mathrm{D}$ value signifies high frequencies of polymorphism indicating decrease in a population size by balancing selection. Tajima's $\mathrm{D}$ value greater than +2 or less than -2 are considered as significant $[59,60]$. In our result we found $\mathrm{D}$ value of 4.904140 which is greater than 0 $(\mathrm{D}>0)$ and +2 (Table 7). This signifies MAPKs are undergone high frequencies of polymorphism by decreasing population size due to balanced selection. So, the heterozygosity of plant MAPKs are more than that of number of segregating sites and presented as multiple alleles.

\section{Duplication of MAPKs}

The size of the plant genome and number of chromosomes in each genome varies widely among species and shows diversity of 2350-folds ranges from 63 to 149,000 $\mathrm{Mb}$ that divided into $\mathrm{n}=2$ to $\mathrm{n}=\sim 600$ chromosomes [25]. Chromosomes evolved by fusion, fission, insertion, and duplication events, allowing evolution of chromosome size and number and hence the genes $[61,62]$. Vascular plants evolved approximately 410 million years ago and diverged into several lineages, among which lycophytes, ferns and gymnosperm and seed plant survived [63]. The transitions from aquatic to land plants, gametophytic generation to sporophytic generation, non-vascular form to vascular form and non seed bearing to seed bearing life cycles requires evolution of new genes $[63,64]$. The co-linearity resulting from the common ancestry of angiosperms provides a powerful method to determine the orthology [65-67]. The MAPK genes from diverse species investigated in this study were found to be orthologous. Based on the results of this study, the orthologous MAPK genes have undergone duplications and given rise to several paralogous genes. The MAPKs those contained novel activation loop motif were found to be duplicated (Table 8). The most common MAPK (AtMPK1) that contain classical T-E-Y motif in its activation loop motif is highly duplicated with z-score 19.46 and 100\% confidence level. The AtMPK20 that contained T-D-Y motif in its activation loop motif resulted in z-score of 11.88 with $100 \%$ confidence interval. All the newly identified MAPKs that contained novel activation loop motifs resulted in z-score more than four with $100 \%$ confidence level. Genes with zscore value four or more than four were considered as highly significant to be duplicated [68]. The phylogenetic study showed that MAPK genes of the grass family of monocotyledonous plants (O. sativa, S. bicolor, B. distachyon, $P$. virgatum, S. italica and $Z$. mays) are more conserved and clustered together, demonstrating that grass 
family MAPKs were evolved from a single lineage. It has been reported that, although genomes of the grass family differ greatly in terms of size, ploidy level and chromosome number, their genetic markers and genes are very well conserved between genomes [69,70]. It has also been reported that there are several duplicated MAPK genes in grass family plants due to their ploidy level and genome duplication [71,72].

Vascular plants appeared approximately 410 million years ago, then diverged into several lineages [73]. The first non-seed vascular plant, Selaginella, lacks evidence of whole genome duplication or polyploidy, which explain why S. moellendorffii contains only six MAPK genes. Although the genome sizes of S. moellendorffii and A. thaliana are very similar [63], A. thalaiana contains 16,574 (65\%) duplicated genes [62]. The transition from a gametophyte to sporophyte dominant life cycle requires far fewer new genes than the transition from non-vascular non-flowering to vascular and flowering plants [63]. Earlier studies of Chlamydomonas, Physcomitrella, Selaginella and fifteen other angiosperm species by Banks et al., revealed that transition from single celled green algae to multi-cellular land plants requires 3006 new genes, but that transition from non-vascular to vascular plants is associated with a gain of only 516 genes [63]. They also reported that gene and genome duplication is pre-requisite for transition from simple leafless nonvascular sporophyte generation to dominant vascularized gametophyte generation and need almost three times as many new genes. The orthologous Physcomitrella and Selaginella shares around $84 \%$ to $89 \%$ of their genes in angiosperm plants, indicating their role as common ancestors [63]. These findings confirm that development from nonvascular to vascular life requires the stepwise addition of new genes directing their extra role in meristem development and hormonal signaling.

\section{MAPK groups}

In this study, we identified MAPK gene family members from different plant groups including monocots, dicots and lower eukaryotic organisms. Overall, the plants included six monocots, 26 dicots, five algae, one bryophyte, one pteridophyte, and one gymnosperm. The amplification and diversification of the large MAPK family in the monocot plant $P$. virgatum could be largely attributed to its tetraploid nature $(2 n=4 \times=36)$ [17]. It will be interesting to determine whether the paralogs in a single group have attained diverse functions in P. virgatum. Among the studied monocots, S. bicolour, S. italica and B. distachyon were found to have 16 member MAPK families. Similar numbers of MAPKs in Brachypodium have been reported by Chen et al. [18].

Group A MAPKs of monocot plants are very stable orthologs of both AtMPK3 and AtMPK6, whereas none of any monocot genomes harbor orthologs of AtMPK10, suggesting that this MAPK may have been lost before the split of monocots. Group B MAPKs of monocot plants posses several paralogs of MPK4, suggesting that the duplication of MPK4 paralogs might have occurred before divergence of monocot plants. Group C MAPKs in monocots contain paralogs of MPK7 and MPK14, but lack any MAPK1 and MAPK2 gene (Table 5). However, a recent study reported the presence of ZmMPK1 and ZmMPK2 in maize, which are putative orthologs of AtMPK1 and AtMPK2, respectively [15]. Liu et al. showed that ZmMPK1 and ZmMPK2 are phylogenetically closer to AtMPK1 and AtMPK2 than AtMPK7 and AtMPK14, respectively, and thus suggested that naming these MAPKs as ZmMPK1 and ZmMPK2 would be more appropriate. This group C MAPK was previously named as ZmMPK7 [74]. However, evaluation of the evolutionary relationship of monocot MAPKs indicated that it would be more appropriate to name these MAPKs as ZmMPK7 and ZmMPK14. Group D MAPKs of monocot plants showed the presence of 10/11 MAPK genes and are relatively constant as compared to the 15 MAPKs in $P$. virgatum (Table 1). These findings indicate that the amplification of monocot group D MAPKs occurred before diversification of this species.

Group A MAPKs, which are relatively constant in monocots with two or four members and in dicot plants, showed zero to seven MAPK members. M. guttatus, which is phylogenetically closer to Arabidopsis, do not possess any group A MAPKs (Table 5). Another plant, potato (S. tuberosum), is particularly conspicuous in that it lacks the group A MAPK from its genome. S. lycopersicum, another member of solanaceae family, has three group A MAPKs in its genome. The absence of complete group A MAPKs in M. guttatus and S. tuberosum is intriguing, and more work in these plant systems is needed to reveal whether other groups of MAPKs have acquired many intrinsic functions of group A MAPKs in these plant species. Another member of group A, an ortholog of AtMPK10, was only observed in B. rapa, T. halophila and C. rubella, which all belong to the mustard family. These findings suggest that MPK10 orthologs are conserved only in the brassicaceae family of dicots, while it was lost from other families (except Selaginella moelendorffii and Picea abies) during evolution (Table 5).

The solanaceous plant S. tuberosum lacks group A MAPKs from their genome, suggesting that they have recently lost this group of MAPKs. The absence of group A MAPKs is particularly intriguing as it comprises orthologs of Arabidopsis MPK3 and MPK6. These two MAPKs show a high level of basal expression and even higher expression in response to biotic and abiotic stress, not only in Arabidopsis, but also in other plants. The roles of these two MAPKs in plant development have been well established in stomatal patterning [75], ovule development [76], 
seed formation and modulation of primary and lateral root development [77]. Definitive functions of group A MAPKs in growth and development and responses to biotic and abiotic stress have been shown in other plants as well [6,10,78-81]. Furthermore, double mutants of mpk3 mpk6 show embryo lethality [10]. These studies in different plant systems suggest that MPK3 and MPK6 orthologs in different plant species are indispensable. It will be interesting to study these plants to address basic questions regarding MAPK signaling in response to different stress and developmental signals. Orthologs of AtMPK10 are confined to the dicot species B. rapa, T. halophila and C. rubella, which all belong to the mustard family. These findings suggest that MPK10 orthologs are only conserved in the brassicaceae family of dicots and have been lost from other families in the course of evolution (Table 5). The presence of four BrMPK10 paralogs might be due to duplication after $B$. rapa speciation since other mustard family members have single orthologs of MPK10. Similar to MPK3 and MPK6 orthologs, group A MAPKs are either present together or absent. The paralogs of MPK3 and MPK6 have been reported in several plant species. A recent report identified MAPKs in canola (B. napus) shows absence of MPK10 in its genome [16]. It is interesting to note that $B$. napus (AACC) is an allotetraploid between B. rapa (AA) and B. oleracea (CC). Evaluation of the expression using the ATH1 GeneChip (at Arabidopsis thaliana Kinase Database-AthKD) under control and different abiotic stress conditions revealed significant expression of AtMPK10 only in three stages/tissues from 208 tissues/stress conditions. These findings suggest a limited role of AtMPK10 in Arabidopsis. However, it will be interesting to investigate the functional relevance of paralogs of MPK10 in B. rapa. For group B MAPKs, with the exception of $A$. thaliana, no orthologs of AtMPK11 was observed in any of the studied dicot plant species, even in the mustard family (Table 5). In Arabidopsis, AtMPK11 showed significant expression in several tissues and under various stress conditions, suggesting it plays a specific role and will not likely to be lost or evolve into a pseudogene. Similarly, MAPK5, another group B MAPK is only restricted to brassicaceae family members with the exception of being present in S. lycopersicum. Eight of ten group B MAPKs in G. max are paralogs of MAPK4. Such a high number of paralogs in soybean suggests extensive events of duplication might have occurred in its genome or part of the genome. These findings are concurrent with recent reports that showed G. $\max$ underwent at least two putative genome wide and/or segmental duplications approximately 13 and 59 million year ago [82-86].

The group C MAPKs members in dicot plants were ranges from one to six (Table 5). The single members of the MAPK group were found in three dicot species, E. grandis, M. guttatus and C. papaya, in the form of either MPK7 or MPK1. The four orthologs of Arabidopsis (AtMPK1, AtMPK2, AtMPK7, and AtMPK14) are not restricted to a particular family or species of dicot plants. The group D MAPKs are unique owing to their 'TDY' motif in the activation loop and long $\mathrm{C}$ terminal common docking domain (Rodriguez et al., 2010 [10]). In dicot plants, the number of group D MAPKs varies from 2 in $A$. coerulea to 14 MAPKs in B. rapa. Among the studied dicot species, 21 showed less than ten group D MAPKs, suggesting that amplification of this group of MAPKs is more prominent in monocots.

We previously discussed loss of group A MAPK MPK10 from monocots and all dicots except members of the brassicaceae family. The lower eukaryotic plant Selaginella and gymnosperm plant Picea abies were found to contain MPK10, a group A MAPK. These findings suggested that MPK10 were existed in older species and are lost during divergence and speciation, after which it was only able to be transferred to members of the brassicaceae family. To identify the evolutionary path of MAPKs, Doczi et al., analyzed MAPK signaling components in evolutionarily representative species of a plant lineage from a free-living amoebaflagellate protist, Naegleria (representative of an early diverging eukaryotic clade, Heterolobosea) to moss, algae and lycophytes [20]. Their study reported the presence of a single conventional MAPK in Naegleria with a 'TEY' motif, suggesting that the common ancestor of MAPKs were ERK-like, from which the distinct classes of MAPKs having TxY signature motifs were diverged [20]. Our study also showed multiple MAPKs (TEY, 2/3/4 and TDY, 1/2) in unicellular and multi-cellular algae (Table 5), suggesting that diversification events of MAPKs started in very early periods of the evolution of photosynthetic eukaryotes.

As the complexity of organisms increased, the MAPK family showed expansion via gene duplication [20]. The $P$. patens, a model moss species with a genome of $487 \mathrm{Mb}$ [87], contains eight MAPKs, six of which were TEY type and two were TDY type (Table 5). The six TEY MAPKs were belonged to group B (four MAPKs) and group $C$ (two MAPKs). Thus, on the evolutionary path of photosynthetic eukaryotes, lycophyetes, moss, multicellular and unicellular algae shows both TEY and TDY types of MAPKs. These findings suggest that the differences between these two types of MAPKs are ancient. Among mosses and algae, P. patens and O. lucimarinus lack 'group A' type MAPKs, while $V$. cartieri lacks 'group B' and 'group C' MAPKs, and C. reinhardtii and C. subellipsoidea do not possess 'group C' MAPKs. Interestingly, none of the studied species lacked 'group D' MAPKs. This observation suggests that the group D MAPKs or TDY MAPKs are indispensable in the lineage of green plants. 
Overall, the MAPK gene family appears to be relatively constant in monocots (with the exception of $P$. virgatum), but not in dicots. Overall, the largest and smallest MAPK genes were observed in dicot plants.

\section{Conclusion}

Genome wide analysis of MAPK gene family revealed presence of new activation loop motifs. The adopted MAPK nomenclature system can be extended to other plant species to maintain uniformity in the MAPK nomenclature system. Presence of novel activation loop motifs are new variants and could offer new type of gene regulation in plants.

\section{Methods}

\section{Identification of MAPK gene family members}

Mitogen activated protein kinase (MAPK) gene families from the model plant Arabidopsis thaliana were downloaded from The Arabidopsis Information Resources (TAIR: http://www.arabidopsis.org/) database [88]. The MAPK gene families from rice were downloaded from the TIGR rice Genome Annotation Resources (http:// rice.plantbiology.msu.edu/) database [89]. The protein sequences of MAPKs from Arabidopsis thaliana and rice were used as search queries in the publicly available phytozome database (http://www.phytozome.net/) to identify MAPK genes in other plant species [90]. Overall, 40 species were included in this study and reported in Table 1 . To identify MAPK gene families of unknown species, BLASTP searches was conducted using orthologous protein sequences Arabidopsis thaliana and Oryza sativa MAPK genes as the query search [91]. The genes identified through BLAST searches were used for further analysis. First, the top 100 genes were kept for systemic evaluation and indexing. The genes with serine/threonine protein kinase domains and the activation loop T-E-Y or T-D-Y motifs were considered as probable MAPK genes, which were subsequently confirmed by scanning in scan prosite and smart software for the presence of MAPK domain $[92,93]$. All datas were checked for redundancy and no any alternative splice variants were considered. Identified MAPK gene families from each species were again confirmed by running BLASTP searches against TAIR using the default parameters $[92,93]$. The genes were considered MAPK genes when BLASTP search matches with Arabidopsis MAPKs.

\section{Multiple sequence alignment and construction of quaternary structure}

Multiple sequence alignment of MAPKs were carried out using the multiple sequence alignment tool Multalin (http://multalin.toulouse.inra.fr/multalin/) to identify the conserved domains. The following default parameters were used to run the multiple alignment programs: Multalin: fasta, protein weight matrix: Blosum62-12-12, gap penalty at opening: default, gap penalty at extension: default, gap penalties at extremities: none, one iteration only: no, high consensus value: $90 \%$ (default), low consensus value: $50 \%$ (default), maximum line length: 180 , and graduation step: 10. Molecular structures of AtMPK1 (group C), SiMPK20-1 (group D) and other MAPKs those contain novel activation loop motif were constructed by Phyre [94] software and resulted PDB file was subjected PYMOL to indicate activation loop motif of MAPKs.

\section{Construction of phylogenetic tree}

To construct the phylogenetic tree, all protein sequences were used to prepare a clustal file by running MAPKs protein sequences in clustal omega software using the following default parameters [95]: output format: clustal w/o numbers, dealign input sequences: no, MBED-like clustering guide tree: yes, MBED-like clustering iteration: yes, number of combined iterations: default (0), maximum guide tree iterations: default, maximum HMM (hidden Markov model) iterations: default, order: aligned. The generated clustal file of MAPKs was converted to MEGA file (.meg) format using the MEGA6 software and then employed to construct a phylogenetic tree [96]. The statistical parameters used to construct the phylogenetic tree were: analysis: phylogeny reconstruction; statistical method: maximum likelihood; test of phylogeny: bootstrap method; no. of bootstrap replications: 2000; substitution type-amino acids; model/method: JonesTaylor-Thornton (JTT) model; rates among sites: uniform rates; gaps/missing data treatment: partial deletion; site coverage cutoff: $95 \%$; ML heuristic method: nearest-neighbor-interchange (NII); initial tree for ML: make initial tree automatically (default-NJ/BioNJ); branch swap filter: very strong.

\section{Statistical analysis}

Tajima's relative rate test was carried out to understand the statistical significance between three different plant groups. The MEGA file used to construct phylogenetic tree was subjected to analyze Tajimas's relative rate test. Random MAPK sequences were selected to analyze Tajima's relative rate test in MEGA6 software. This random analysis was replicated for two times. Different statistical parameters used to analyze Tajima's relative rate test were; scope: for three chosen sequence, substitution type: amino acid gaps/ missing data treatment: complete deletion. On the other hand, Tajima's test of neutrality was carried out to understand the evolution of randomly evolved MAPKs from none randomly evolved MAPKs. Different statistical parameters used to analyze Tajima's test of neutrality were; scope: all 
selected taxas, substitution type: amino acid, gaps/missing data treatment: complete deletion.

The overall mean distances of all the MAPKs were calculated using MEGA6 software. The MEGA file used to construct phylogenetic tree was used to analyze the overall mean distance of plant MAPKs. Different statistical parameters used to calculate the overall mean distances were; analysis: distance estimation, scope: overall mean, variance estimation method: bootstrap method, no. of boot strap replication: 2000, substitution type: amino acid, model/method: p-distance, rate among site: uniform, pattern among lineage: same (homogenous), gaps/missing data treatment: partial deletion.

\section{Nomenclature of MAPKs}

All predicted MAPKs were named based on the evolutionary relationship of MAPKs with Arabidopsis thaliana or Oryza sativa MAPKs as suggested by Hamel et al. [21]. During the nomenclature process, the first letter in upper case was used to identify the genus name, the second letter in lower case (in a few cases the first 2-3 letters) was used to identify species name, after which the MPK and number of corresponding orthologs of Arabidopsis or Oryza sativa. The monocot plant species were named according to the MAPK ortholog of Oryza sativa, while other species were named according to MAPK orthologs of Arabidopsis thaliana. If more than one ortholog was present in a particular species (or in the case of paralogs), the second number is followed by a hyphen to distinguish between paralogs.

\section{Additional files}

\section{Additional file 1: Additional data file showing the unified nomenclatured gene name, locus ID and detailed genomic information of plant MAPKs.}

Additional file 2: Additional data file showing the MAPK gene name, molecular weight (in $\mathrm{kDa}$ ) and predicted isoelectric ( $\mathrm{pl}$ ) point.

Additional file 3: Additional data file showing the average amino acid composition of plant MAPKs.

Additional file 4: Additional data file showing multiple sequence alignment of all studied MAPK genes of $\mathbf{4 0}$ different plant species. Additional file 5: Additional data file showing the phylogenetic tree of plant MAPKs. Red color indicates group A, blue color group B, pink color group C, purple color group D, teal color group $E$ and green color represent group F MAPKs.

\section{Abbreviation}

MAPK: Mitogen activated protein kinase; pl: Isoelectric point; kDa: Kilo dalton.

\section{Competing interests}

The authors declare that they have no competing interests.

\section{Authors' contributions}

TKM: Conception and design of the experiments, sequence and statistical analysis, interpretation of data's, prepared the manuscript draft. PKA: Analyzed the data, prepared the manuscript draft. NM: Performed the experiments, data analysis, drafted the manuscript. PP: Modeled the molecular structures of MAPKs. HB: Revised the manuscript and given approval for publication. All authors read and approved the final manuscript.

\section{Acknowledgements}

Authors also like to say thanks to Dr. Alok Krishna Sinha, staff scientist, National Institute of Plant Genome Research, New Delhi, India and Dr. Dhammaprakash Wankhede, National Bureau of Plant Genetic Resources, New Delhi, India for their constructive and valuable suggestions in the manuscript. This work was carried out with the support of the "Cooperative Research Program for Agriculture Science \& Technology Development (PJ01049704)" Rural Development Administration, Republic of Korea. The funders had no role in study design, data collection and analysis, decision to publish, or preparation of the manuscript.

\section{Author details}

'School of Biotechnology, Yeungnam University, Daehak Gyeongsan, Gyeonsangbook 712749, Republic of Korea. ${ }^{2}$ Department of Biotechnology, North Orissa University, Sri Ramchandra Vihar, Takatpur, Baripada, Mayurbhanj, Orissa 757003, India. ${ }^{3}$ Center for Studies in Biotechnology, Dibrugarh University, Dibrugarh, Assam 786004, India.

Received: 27 November 2014 Accepted: 15 January 2015 Published online: 06 February 2015

\section{References}

1. Ellis BE. Postal code for a plant MAPK. Biochem J. 2012;446:e5-7.

2. Besteiro MAG, Ulm R. Phosphorylation and Stabilization of Arabidopsis MAP Kinase Phosphatase 1 in Response to UV-B Stress. J Biol Chem. 2013;288:480-6.

3. Caunt CJ, Keyse SM. Dual-specificity MAP kinase phosphatases (MKPs) Shaping the outcome of MAP kinase signalling. FEBS J. 2013;280:489-504.

4. Hanks SK. Genomic analysis of the eukaryotic protein kinase superfamily: a perspective. Genome Biol. 2003;4:111.

5. Li S, Franklin-Tong V. Modulating and monitoring MAPK activity during programmed cell death in pollen. Methods Mol Biol. 2011;779:165-83.

6. Sinha AK, Jaggi M, Raghuram B, Tuteja N. Mitogen-activated protein kinase signaling in plants under abiotic stress. Plant Signal Behav. 2011;6:196-203.

7. Meldau S, Ullman-zeunert L, Govind G, Bartram S, Baldwin IT. MAPKdependent JA and SA signalling in Nicotiana attenuata affects plant growth and fitness during competition with conspecifics. BMC Plant Biol. 2012;12:1-15.

8. Shen H, Liu C, Zhang Y, Wang X. OsWRKY30 is activated by MAP kinases to confer drought tolerance in rice. Plant Mol Biol. 2012;80:241-53.

9. Cheong YH, Kim K, Pandey GK, Gupta R, Grant JJ, Luan S. CBL1, a calcium sensor that differentially regulates salt, drought, and cold responses in Arabidopsis. Plant Cell. 2003;15:1833-45.

10. Rodriguez MC, Petersen M, Mundy J. Mitogen-Activated Protein Kinase signaling in plants. Annu Rev Plant Biol. 2010;61:621-49.

11. Steichen JM, Kuchinskas M, Keshwani M, Yang J, Adams JA, Taylor SS. Structural Basis for the Regulation of Protein Kinase A by Activation loop Phosphorylation. J Biol Chem. 2012:287:14672-80.

12. Deleris P, Rousseau J, Coulombe P, Rodier G, Tanguay P, Meloche S. Activation Loop Phosphorylation of the Atypical MAP Kinases ERK3 and ERK4 Is Required for Binding, Activation and Cytoplasmic Relocalization of MK5. J Cell Physiol. 2008;217:778-88.

13. Ichimura(MAPK Group) K, Shinozaki K, Tena G, Sheen J, Henry Y, Champion $A$, et al. Mitogen-activated protein kinase cascades in plants: a new nomenclature. Trends Plant Sci. 2002;7:301-8.

14. Reyna NS, Yang Y. Molecular Analysis of the Rice MAP Kinase Gene Family in Relation to Magnaporthe grisea Infection. Mol plant-microbe Interact. 2006;19:530-40.

15. Liu Y, Zhang D, Wang L, Li D. Genome-Wide Analysis of Mitogen-Activated Protein Kinase Gene Family in Maize. Plant Mol Biol Report. 2013;31:1446-60.

16. Liang W, Yang B, Yu B-J, Zhou Z, Li C, Jia M, et al. Identification and analysis of MKK and MPK gene families in canola (Brassica napus L.). BMC Genomics. 2013;14:392

17. Zhang S, Xu R, Luo X, Jiang Z, Shu H. Genome-wide identification and expression analysis of MAPK and MAPKK gene family in Malus domestica. Gene. 2013;531:377-87. 
18. Chen L, Hu W, Tan S, Wang M, Ma Z, Zhou S, et al. Genome-wide identification and analysis of MAPK and MAPKK gene families in Brachypodium distachyon. PLoS One. 2012;7:e46744

19. Janitza P, Ullrich KK, Quint M. Toward a comprehensive phylogenetic reconstruction of the evolutionary history of mitogen-activated protein kinases in the plant kingdom. Front Plant Sci. 2012;3:1-11.

20. Dóczi R, Okrész L, Romero AE, Paccanaro A, Bögre L. Exploring the evolutionary path of plant MAPK networks. Trends Plant Sci. 2012;17:518-25.

21. Hamel L-P, Nicole M-C, Sritubtim S, Morency M-J, Ellis M, Ehlting J, et al. Ancient signals: comparative genomics of plant MAPK and MAPKK gene families. Trends Plant Sci. 2006;11:192-8.

22. Lin J, Gerstein M. Whole-genome trees based on the occurrence of folds and orthologs: implications for comparing genomes on different levels. Genome Res. 2000;10:808-18.

23. Remm M, Storm CEV, Sonnhammer ELL. Automatic clustering of orthologs and in-paralogs from pairwise species comparisons. J Mol Biol. 2001:314:1041-52.

24. Aravind L, Koonin EV. Towards understanding the first genome sequence of a crenarchaeon by genome annotation using clusters of orthologous groups of proteins ( COGs ) Darren A Natale, Uma T Shankavaram, Michael Y Galperin, Yuri I Wolf. Genome Biol. 2000;1:1-19.

25. Heslop-Harrison JSP, Schwarzacher T. Organisation of the plant genome in chromosomes. Plant J. 2011;66:18-33.

26. Heslop-Harrison JS. Comparative genome organization in plants: from sequence and markers to chromatin and chromosomes. Plant Cell. 2000;12:617-36.

27. De KK, Saha A, Tamang R, Sharma B. Investigation on relative genome sizes and ploidy levels of Darjeeling-Himalayan Rhododendron species using flow cytometer. Indian J Biotechnol. 2010;9:64-8.

28. Ohri D. Genome Size Variation and Plant Systematics. Ann Bot. 1998:82:75-83.

29. Parris JK, Ranney TG, Baird W. Ploidy levels, relative genome sizes, and base pair composition in magnolia. J Amer Soc Hort Sci. 2010;135:533-47.

30. Shearer K, Ranney TG, Crop M, Crops MH, River M. Ploidy level and relative genome size of species, hybrids and cultivars of Dogwood (Cornus spp ). HortScience. 2013;48:825-30.

31. Altenhoff AM, Studer RA, Robinson-Rechavi M, Dessimoz C. Resolving the ortholog conjecture: orthologs tend to be weakly, but significantly, more similar in function than paralogs. PLoS Comput Biol. 2012:8:e1002514.

32. Mattick J. Introns: evolution and function. Curr Opin Genet Dev. 1994;4:823-31.

33. Tyanova S, Cox J, Olsen J, Mann M, Frishman D. Phosphorylation variation during the cell cycle scales with structural propensities of proteins. PLoS Comput Biol. 2013;9:e1002842.

34. Cohen P. The origins of protein phosphorylation. Nat Cell Biol. 2002;4:E127-30.

35. Román E, Pla J. The CEK1-mediated mitogen-activated protein kinase pathway in the fungal pathogen Candida albicans. MAP Kinase. 2013;2:e5.

36. Guo X, He D, Huang L, Liu L, Liu L, Yang H. Strain energy in enzyme-substrate binding: An energetic insight into the flexibility versus rigidity of enzyme active site. Comput Theor Chem. 2012;995:17-23.

37. Wang L, Althoff EA, Bolduc J, Jiang L, Moody J, Lassila JK, et al. Structural analyses of covalent enzyme-substrate analog complexes reveal strengths and limitations of de novo enzyme design. J Mol Biol. 2012;415:615-25.

38. Miller BG. The mutability of enzyme active-site shape determinants. Protein Sci. 2007;16:1965-8.

39. Peden EA, Boehm M, Mulder DW, Davis R, Old WM, King PA, et al. Identification of global ferredoxin interaction networks in Chlamydomonas reinhardtii. J Biol Chem. 2013;288:35192-209.

40. Lise S, Walker-Taylor A, Jones DT. Docking protein domains in contact space. BMC Bioinformatics. 2006;7:310.

41. Tanoue T, Adachi M, Moriguchi T, Nishida E. A conserved docking motif in MAP kinases common to substrates, activators and regulators. Nat Cell Biol. 2000;2:110-6.

42. Lee $\mathrm{S}$, Lin X, Nam NH, Parang K, Sun G. Determination of the substratedocking site of protein tyrosine kinase C-terminal Src kinase. Proc Natl Acad Sci U S A. 2003;100:14707-12

43. Xie $\mathrm{Q}$, Joseph RE, Fulton $\mathrm{DB}$, Andreotti $\mathrm{AH}$. Substrate recognition of $\mathrm{PLC} 1$ via a specific docking surface on Itk. J Mol Biol. 2013;425:683-96.

44. Lee S, Ayrapetov MK, Kemble DJ, Parang K, Sun G. Docking-based substrate recognition by the catalytic domain of a protein tyrosine kinase, C-terminal Src kinase (Csk). J Biol Chem. 2006;281:8183-9.

45. Hamel L-P, Sheen J, Séguin A. Ancient signals: comparative genomics of green plant CDPKs. Trends Plant Sci. 2014;19:79-89.
46. Edgell DR, Malik SB, Doolittle WF. Evidence of independent gene duplications during the evolution of archaeal and eukaryotic family B DNA polymerases. Mol Biol Evol. 1998;15:1207-17.

47. Cock JM, Sterck L, Rouze P, Scornet D, Allen AE, Amoutzias G, et al. The Ectocarpus genome and the independent evolution of multicellularity in brown algae. Nature. 2010:465:617-21.

48. Ohta T. Evolution of gene families. Gene. 2000;259:45-52.

49. Zhao Z-M, Reynolds AB, Gaucher EA. The evolutionary history of the catenin gene family during metazoan evolution. BMC Evol Biol. 2011;11:198.

50. Levine MT, McCoy C, Vermaak D, Lee YCG, Hiatt MA, Matsen FA, et al. Phylogenomic analysis reveals dynamic evolutionary history of the Drosophila heterochromatin protein 1 (HP1) gene family. PLoS Genet. 2012:8:e1002729.

51. Demuth JP, De Bie T, Stajich JE, Cristianini N, Hahn MW. The evolution of mammalian gene families. PLoS One. 2006;1:e85

52. Lima MDF, Eloy NB, Pegoraro C, Sagit R, Rojas C, Bretz T, et al. Genomic evolution and complexity of the Anaphase-promoting Complex (APC) in land plants. BMC Plant Biol. 2010;10:254

53. Lan T, Yang Z-L, Yang X, Liu Y-J, Wang X-R, Zeng Q-Y. Extensive functional diversification of the Populus glutathione S-transferase supergene family. Plant Cell. 2009;21:3749-66.

54. Zhao S, Liang Z, Demko V, Wilson R, Johansen W, Olsen O-A, et al. Massive expansion of the calpain gene family in unicellular eukaryotes. BMC Evol Biol. 2012;12:193.

55. Arabidopsis T, Initiative G. Analysis of the genome sequence of the flowering plant Arabidopsis thaliana. Nature. 2000;408:796-815.

56. Kim J, Shiu S-H, Thoma S, Li W-H, Patterson SE. Patterns of expansion and expression divergence in the plant polygalacturonase gene family. Genome Biol. 2006;7:R87.

57. Lespinet $\mathrm{O}$, Wolf $\mathrm{Yl}$, Koonin $\mathrm{EV}$, Aravind $\mathrm{L}$. The role of lineage-specific gene family expansion in the evolution of eukaryotes. Genome Res. 2002;12:1048-59.

58. Yang X, Kalluri UC, Jawdy S, Gunter LE, Yin T, Tschaplinski TJ, et al. The F-box gene family is expanded in herbaceous annual plants relative to woody perennial plants. Plant Physiol. 2008;148:1189-200.

59. Tajima F. Statistical Method for Testing the Neutral Mutation Hypothesis by DNA Polymorphism. Genetics. 1989;595:585-95.

60. Tajima F. Simple Methods for Testing the Molecular Evolutionary Clock Hypothesis. Genetics. 1993;135:599-607.

61. Bowers JE, Chapman BA, Rong J, Paterson AH. Unravelling angiosperm genome evolution by phylogenetic analysis of chromosomal duplication events. Nature. 2003;422:433-8.

62. Zhang J. Evolution by gene duplication: an update. Trends Ecol Evol. 2003;18:292-8

63. Banks JA, Nishiyama T, Hasebe M, Bowman JL, Gribskov M, DePamphilis C, et al. The Selaginella genome identifies genetic changes associated with the evolution of vascular plants. Science. 2011;332:960-3.

64. Aubret F, Bonnet $X$, Shine $R$. The role of adaptive plasticity in a major evolutionary transition: early aquatic experience affects locomotor performance of terrestrial snakes. Funct Ecol. 2007;21:1154-61.

65. Dewey CN. Positional orthology: putting genomic evolutionary relationships into context. Brief Bioinform. 2011;12:401-12

66. Gabaldon T, Koonin EV. Functional and evolutionary implications of gene orthology. Nat Rev Genet. 2013;14:360-6.

67. Paterson A, Freeling M, Tang H, Wang X. Insights from the Comparison of Plant Genome Sequences. Annu Rev Plant Biol. 2010;61:349-72.

68. Kontopoulos DG, Glykos NM. Pinda: a web service for detection and analysis of intraspecies gene duplication events. Comput Methods Programs Biomed. 2013;111:711-4.

69. Feuillet C, Keller B. Comparative genomics in the grass family: molecular characterization of grass genome structure and evolution. Ann Bot. 2002;89:3-10.

70. Eckardt N. Grass genome evolution. Plant Cell Online. 2008;20:3-4.

71. Caffrey DR, O'Neill LA, Shields DC. The evolution of the MAP kinase pathways: coduplication of interacting proteins leads to new signaling cascades. J Mol Evol. 1999:49:567-82.

72. Li M, Liu J, Zhang C. Evolutionary history of the vertebrate mitogen activated protein kinases family. PLoS One. 2011;6:e26999.

73. Raven JA, Edwards D. Roots: evolutionary origins and biogeochemical significance. J Exp Bot. 2001;52(Spec Issue):381-401.

74. Zong XJ, Li DP, Gu LK, Li DQ, Liu LX, Hu XL. Abscisic acid and hydrogen peroxide induce a novel maize group C MAP kinase gene, ZmMPK7, 
which is responsible for the removal of reactive oxygen species. Planta. 2009;299:485-95.

75. Wang H, Ngwenyama N, Liu Y, Walker JC, Zhang S. Stomatal development and patterning are regulated by environmentally responsive mitogenactivated protein kinases in Arabidopsis. Plant Cell. 2007;19:63-73.

76. Wang H, Liu Y, Bruffett K, Lee J, Hause G, Walker JC, et al. HaploInsufficiency of MPK3 in MPK6 Mutant Background Uncovers a Novel Function of These Two MAPKs in Arabidopsis Ovule Development. Plant Cell. 2008;20:602-13.

77. López-Bucio JS, Dubrovsky JG, Raya-González J, Ugartechea-Chirino Y, López-Bucio J, de Luna-Valdez LA, et al. Arabidopsis thaliana mitogenactivated protein kinase 6 is involved in seed formation and modulation of primary and lateral root development. J Exp Bot. 2014;65:169-83.

78. Wen J, Oono K, Imai R, Osmek R. Two novel mitogen-activated protein signaling components, OsMEK1 and OsMAP1, are involved in a moderate low-temperature signaling pathway in rice 1. Plant Physiol. 2002;129:1880-91.

79. Rao KP, Vani G, Kumar K, Wankhede DP, Misra M, Gupta M, et al. Arsenic stress activates MAP kinase in rice roots and leaves. Arch Biochem Biophys. 2011;506:73-82.

80. Raina SK, Wankhede DP, Jaggi M, Singh P, Jalmi SK. CrMPK3, a mitogen activated protein kinase from Catharanthus roseus and its possible role in stress induced biosynthesis of monoterpenoid indole alkaloids. BMC Plant Biol. 2012;12:1.

81. Raina SK, Wankhede DP, Sinha AK. Catharanthus roseus mitogen-activated protein kinase 3 confers UV and heat tolerance to Saccharomyces cerevisiae (c) 2013 Landes Bioscience. Do not distribute (c) 2013 Landes Bioscience. Do not distribute. Plant Signal Behav. 2013;8:116-9.

82. Dong Q, Schlueter SD, Brendel V. PlantGDB, plant genome database and analysis tools. Nucleic Acids Res. 2004;32(Database issue):D354-9.

83. Shoemaker RC, Schlueter J, Doyle JJ. Paleopolyploidy and gene duplication in soybean and other legumes. Curr Opin Plant Biol. 2006;9:104-9.

84. Gill N, Findley S, Walling JG, Hans C, Ma J, Doyle J, et al. Molecular and chromosomal evidence for Allopolyploidy. Plant Physiol. 2009;151:1167-74

85. Lin J, Stupar RM, Hans C, Hyten DL, Jackson SA. Structural and functional divergence of a 1-Mb duplicated region in the soybean (Glycine max) genome and comparison to an orthologous region from Phaseolus vulgaris. Plant Cell. 2010;22:2545-61.

86. Schmutz J, Cannon SB, Schlueter J, Ma J, Thelen JJ, Cheng J, et al. Genome sequence of the palaeopolyploid soybean. Nature. 2010;465:120.

87. Rensing SA, Lang D, Zimmer AD, Terry A, Salamov A, Shapiro $H$ et al.: The Physcomitrella genome reveals evolutionary insight into the conquest of land by plants. Science 2014, 319

88. Lamesch P, Berardini TZ, Li D, Swarbreck D, Wilks C, Sasidharan R, et al. The Arabidopsis Information Resource (TAIR): improved gene annotation and new tools. Nucleic Acids Res. 2012;40:D1202-10.

89. Ouyang S, Zhu W, Hamilton J, Lin H, Campbell M, Childs K, et al. The TIGR Rice Genome Annotation Resource: improvements and new features. Nucleic Acids Res. 2007;35:D883-7.

90. Goodstein DM, Shu S, Howson R, Neupane R, Hayes RD, Fazo J, et al. Phytozome: a comparative platform for green plant genomics. Nucleic Acids Res. 2012;40:D1178-86

91. Altschul SF, Madden TL, Schäffer AA, Zhang J, Zhang Z, Miller W, et al. Gapped BLAST and PSI-BLAST: a new generation of protein database search programs. Nucleic Acids Res. 1997;25:3389-402

92. De Castro E, Sigrist CJA, Gattiker A, Bulliard V, Langendijk-Genevaux PS,

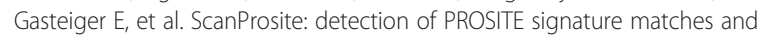
ProRule-associated functional and structural residues in proteins. Nucleic Acids Res. 2006;34:W362-5.

93. Letunic I, Doerks T, Bork P. SMART 7: recent updates to the protein domain annotation resource. Nucleic Acids Res. 2012;40:D302-5.

94. Kelley LA, Sternberg MJE. Protein structure prediction on the Web: a case study using the Phyre server. Nat Protoc. 2009;4:363-71.

95. Sievers F, Wilm A, Dineen D, Gibson TJ, Karplus K, Li W, et al. Fast, scalable generation of high-quality protein multiple sequence alignments using Clustal Omega. Mol Syst Biol. 2011;7:539.

96. Tamura K, Stecher G, Peterson D, Filipski A, Kumar S. MEGA6: Molecular Evolutionary Genetics Analysis version 6.0. Mol Biol Evol. 2013;30:2725-9.

\section{Submit your next manuscript to BioMed Central and take full advantage of:}

- Convenient online submission

- Thorough peer review

- No space constraints or color figure charges

- Immediate publication on acceptance

- Inclusion in PubMed, CAS, Scopus and Google Scholar

- Research which is freely available for redistribution

Submit your manuscript at www.biomedcentral.com/submit 